\title{
Multiple paralogues of a-SNAP in Giardia lamblia exhibit independent subcellular localization and redistribution during encystation and stress
}

\author{
Shankari Prasad Datta ${ }^{1}$, Kuladip Jana ${ }^{2}$, Avisek Mondal ${ }^{1,3}$, Sandipan Ganguly ${ }^{4}$ and Srimonti Sarkar ${ }^{1 *}$ (D)
}

\begin{abstract}
Background: The differently-diverged parasitic protist Giardia lamblia is known to have minimal machinery for vesicular transport. Yet, it has three paralogues of SNAP, a crucial component that together with NSF brings about disassembly of the cis-SNARE complex formed following vesicle fusion to target membranes. Given that most opisthokont hosts of this gut parasite express only one a-SNAP, this study was undertaken to determine whether these giardial SNAP proteins have undergone functional divergence.

Results: All three SNAP paralogues are expressed in trophozoites, encysting trophozoites and cysts. Even though one of them clusters with $Y$-SNAP sequences in a phylogenetic tree, functional complementation analysis in yeast indicates that all the three proteins are functionally orthologous to a-SNAP. Localization studies showed a mostly non-overlapping distribution of these a-SNAPs in trophozoites, encysting cells and cysts. In addition, two of the paralogues exhibit substantial subcellular redistribution during encystation, which was also seen following exposure to oxidative stress. However, the expression of the three genes remained unchanged during this redistribution process. There is also a difference in the affinity of each of these a-SNAP paralogues for GINSF.

Conclusions: None of the genes encoding the three a-SNAPs are pseudogenes and the encoded proteins are likely to discharge non-redundant functions in the different morphological states of $G$. lamblia. Based on the difference in the interaction of individual a-SNAPs with GINSF and their non-overlapping pattern of subcellular redistribution during encystation and under stress conditions, it may be concluded that the three giardial a-SNAP paralogues have undergone functional divergence. Presence of one of the giardial a-SNAPs at the PDRs of flagella, where neither GINSF nor any of the SNAREs localize, indicates that this a-SNAP discharges a SNARE-independent role in this gut pathogen.
\end{abstract}

Keywords: a-SNAP, NSF, SNARE, Oxidative stress, Encystation, Functional divergence, Gene duplication

\section{Background}

Giardia lamblia is a gut pathogen that causes the diarrheal disease giardiasis. In addition, this protist serves as an excellent model to study how eukaryotic evolution has proceeded along different paths [1]. Studies show that the molecular machinery for multiple cellular processes of this protist are highly diverged compared to that present in

\footnotetext{
* Correspondence: srimonti@jcbose.ac.in

${ }^{1}$ Department of Biochemistry, Bose Institute, P 1/12 CIT Road Scheme VII M, Kolkata, West Bengal 700054, India

Full list of author information is available at the end of the article
}

most well-studied model eukaryotes [2]. Whether the differently-diverged cellular pathways of present-day Giardia results from early divergence from the main line of eukaryotic evolution or is a consequence of reductive evolution is still debatable. This divergence is particularly evident in the machinery for maintaining its endomembrane system, which is composed of fewer compartments compared to that present in most eukaryotes [2]. Besides the endoplasmic reticulum (ER), the only other identifiable endomembrane compartments are the small peripheral vesicles (PVs) that discharge both endosomal and 
lysosomal functions [3]. Thus, this parasite is an interesting model to study how vesicular trafficking proceeds in the backdrop of reduced endomembrane compartment diversity and uncovering the molecular machinery that supports this system is important to understand how life has evolved to use different approaches to address the same challenge.

The exchange of material between different endomembrane compartments takes place either through direct contact between the organellar membranes or via transport vesicles [4]. Such exchanges are very precise and sustained by a complex machinery whose molecular components include both proteins and lipids [5]. Vesicle-mediated exchange requires membrane deformation and vesicle budding from donor membrane, transport of these vesicles on the cytoskeletal network, followed by docking at the appropriate target membrane, and finally its fusion to the target membrane [6]. Proteins that ensure the fidelity of this process include: (i) the adapter protein (AP) complex and members of the Sar/Arf GTPases that selectively load appropriate cargo molecules into the budding vesicle and recruit the coat proteins at the donor compartment; (ii) the coat proteins (clathrin, COPI and COPII) that deform the membrane to form the vesicle; and (iii) the Rab GTPases, tether proteins and the SNAREs, which ensure that the vesicles only fuse to the appropriate acceptor compartment [7]. While tether proteins extend out from the acceptor membrane to "scout" for the correct vesicle and bring it closer, the SNAREs operate over a shorter distance and the formation of the trans-SNARE complex brings about the fusion between the membranes of the vesicle and acceptor compartment.

Different members of the SNARE proteins decorate the surfaces of various vesicles and also the target compartments. A trans-SNARE complex is formed when the SNARE on the incoming vesicle precisely pairs-up with the cognate SNAREs on the target membrane. This pairing enables the vesicle and target membranes to come close together such that they can fuse [8]. Following membrane fusion, the helical bundle of the newly-formed cis-SNARE complex is disassembled so that the SNAREs arriving on the incoming vesicle may be recycled back to the donor compartment. The strong intermolecular interactions within the cis-SNARE complexes are broken with the help of a protein complex, consisting of the AAA ATPase N-ethylmaleimide sensitive factor (NSF) and soluble NSF-attachment protein (SNAP), as the energy derived from ATP hydrolysis drives cis-SNARE uncoupling [9].

As previously mentioned, the machinery supporting the endomembrane system of Giardia is composed of fewer components. For example, instead of four, only two AP complexes have been identified in Giardia; in the tethering complexes, three components of both HOPS and TRAPP1, and two components of DSL1 were found to be missing $[10,11]$. Only eight Rab GTPases have been identified in this protist, in contrast to the large repertoire of Rab members in other parasitic protists such as Trichomonas and Entamoeba [12-15]. Even the ESCRT machinery for endosomal sorting is composed of fewer components, with either entire complexes, such as ESCRT-I, being absent, or complexes being composed of fewer subunits, as in the case of ESCRT-II and ESCRT-III [16, 17].

Interestingly, there appear to be exceptions to this observed reduction of cellular machinery, as in the case of SNAPs. Many organisms, including mammals, have multiple paralogues of SNAPs, termed $\alpha$-, $\beta$ - and $\gamma$-SNAPs [18]. In mammals, while $\alpha$ - and $\beta$-SNAPs share a high degree of homology (> 80\% identity), $\gamma$-SNAP shares only $20 \%$ identity with $\alpha$-SNAP. Also, while both $\alpha$ - and $\gamma$-SNAPs are expressed in most tissues, expression of $\beta$-SNAP is restricted to the brain [19]. In addition, expression of $\alpha$-SNAP commences in the developing embryo and continues into adulthood, but $\beta$-SNAP is expressed only after birth [19]. These SNAPs also discharge different cellular functions. $\alpha$-SNAP is required for transport from ER to the Golgi, within the Golgi, homotypic vesicle fusion, store-operated calcium release and ER/endosome membrane fusion [20-22]. $\beta$-SNAP is functionally similar, except it has a different affinity for a binding partner, PICK1 [23]. $\gamma$-SNAP can stimulate the $\mathrm{Ca}^{2+}$-dependent exocytosis but is unable to function in ER to Golgi transport $[24,25]$. While most eukaryotes characterized thus far have only one copy of $\alpha$-SNAP, two $\alpha$-SNAPs have been reported in Giardia [26]. Here we report the presence of not two, but three SNAP genes in the Giardia genome. All the three genes were expressed in trophozoites, encysting trophozoites and cysts. The predicted secondary and tertiary structures of all the three proteins are similar to the yeast $\alpha$-SNAP, Sec17, and all three giardial genes can rescue the growth defect of the sec17-1 temperature-sensitive yeast mutant. Interestingly, there is a distinct difference in the subcellular distribution of all three paralogues. Apart from the anticipated localization at the cell periphery where many membranous compartments are present, two of the paralogues exhibit a dramatic change in subcellular distribution both during encystation and oxidative stress. This observed difference in subcellular distribution is indicative of the paralogues performing distinct functions in this protist.

\section{Results}

Giardia lamblia encodes three paralogues of SNAP

Given that SNAPs play pivotal roles in vesicle-mediated trafficking, we wanted to characterize the previously-reported putative giardial orthologues encoded by the 
ORFs GL50803_17224 and GL50803_16521 [26]. This report also identified the ORF GL50803_10856 as a putative NSF. However, domain analysis in Pfam indicates that like the first two ORFs, the protein encoded by the last also contains a SNAP domain and consistently, GiardiaDB annotates it as an $\alpha$-SNAP. To determine if the proteins encoded by these ORFs have the potential to function as SNAPs, we analyzed their sequence to determine their probable secondary structures. All characterized SNAP orthologues are small $\alpha$-helical proteins, including the 292 residue-long S. cerevisiae orthologue, Sec17 [27]. The three putative giardial SNAPs are

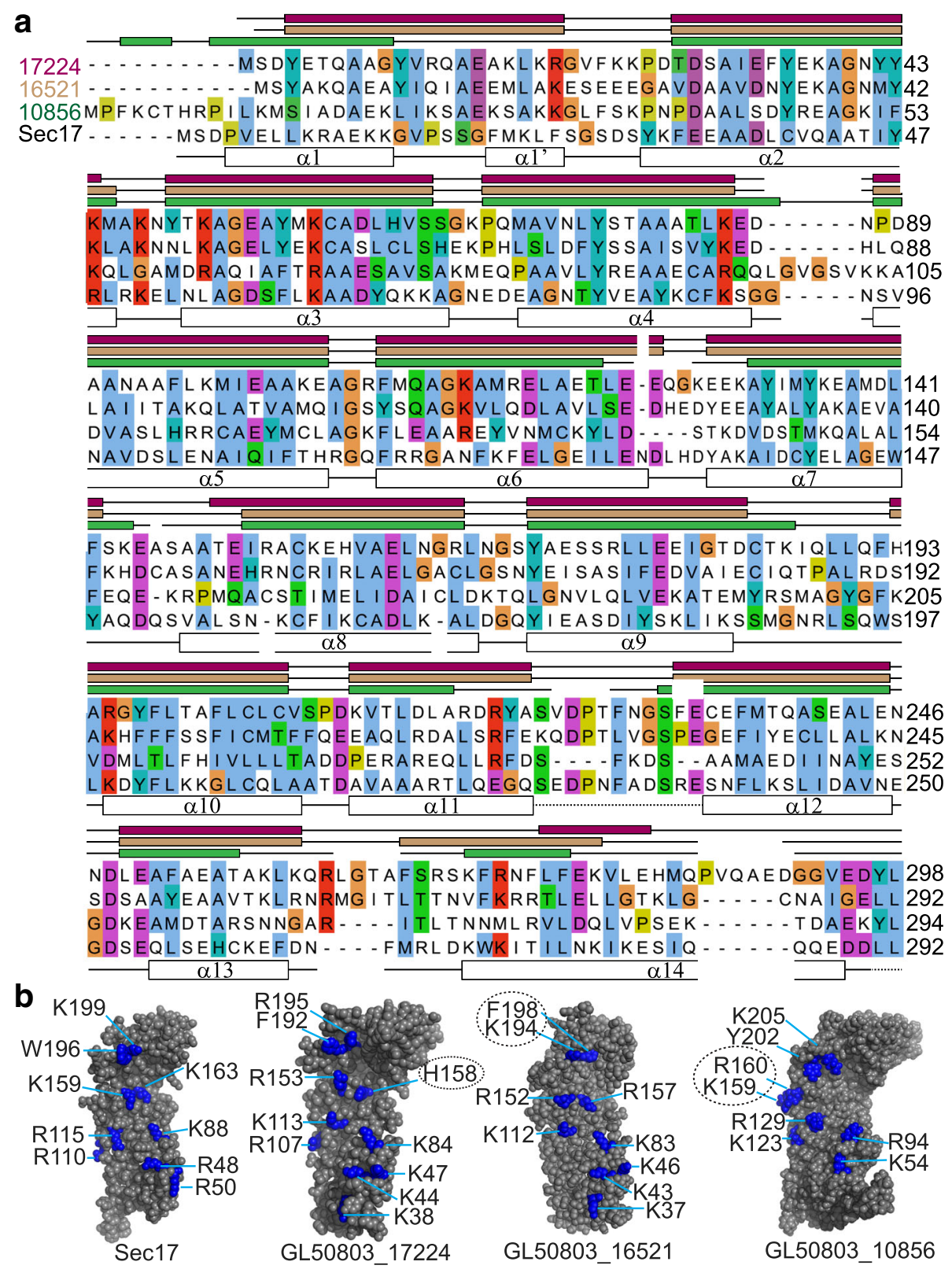

Fig. 1 Secondary structural elements and SNARE-binding residues of giardial SNAPS. a Sequence alignment of the putative giardial SNAPs with Sec17 of S. cerevisiae. a-helical regions of the Sec17 crystal structure (1QQE) have been marked with white boxes below the sequence alignment. The regions of the Giardia SNAPs that are predicted to be a-helical have been marked with colored boxes (SNAP ${ }_{17224}$, cherry; SNAP 16521, fawn; SNAP 10856 , green) above the alignment. Lines indicate loops regions and dashed lines denote the disordered regions in the Sec17 crystal structure. The discontinuity in boxes or lines correspond to gaps in the alignment. $\mathbf{b}$ Residues that may participate in SNARE binding. Residues, mostly carrying a positive charge (see text), which occupy positions comparable to those of the SNARE-binding residues of the $B$. taurus a-SNAP have been mapped in blue onto the concave face of the threading models of both yeast and Giardia SNAPs. Residues that deviate from those of the B. taurus a-SNAP, either in terms of charge (GL50803_17224) or position (GL50803_16521 and GL50803_10856), have been marked with dotted circles 
comparable in size; while GL50803_17224 encodes 298 amino acids, GL50803_16521 and GL50803_10856 are composed of 292 and 294 amino acids, respectively. However, the three ORFs do not share extensive sequence homology; while the sequence of GL50803_17224 is 30.6 and 22.6\% identical to GL50803_16521 and GL50803_10856, respectively, the remaining two ORFs share $21.6 \%$ identity. Secondary structure predictions indicate that all the three putative proteins are composed of $\alpha$-helices and loops (Fig. 1a). This conclusion is independently supported by circular dichroism spectroscopy, which indicates that the percentage helicity of the three proteins is comparable to Sec17 (Additional file 1: Figure S1). Consistent with the crystal structure of Sec17, the three giardial proteins are predicted to have fourteen $\alpha$-helices each (Fig. 1a) [27]. One notable difference is that while in the Sec17 crystal structure, the membrane-interacting hydrophobic patch, GFMKLF, adopts a short $\alpha$ turn $\left(\alpha-1^{\prime}\right)$, the hydrophobic residues in the corresponding region of all the three putative giardial orthologues are located in an unstructured loop between helices $\alpha 1$ and $\alpha 2$ [28]. Thus, all the three giardial proteins are like Sec17 both in terms of their size and the secondary structure they are likely to adopt.

A previous report had identified residues on the SNARE-binding surface of the Bos taurus $\alpha$-SNAP, of which all but one (Y200) were charged [29]. Perusal of the crystal structure of Sec17 showed that almost all the analogous positions are also occupied by similar residues, including W196 occupying a position comparable to Y200 (Fig. 1b). Given the low sequence identity of the three giardial proteins with known SNAP orthologues (Additional file 2: Tables S1 and S2), we wanted to determine their possible tertiary structures to see if similarly-charged amino acid residues occupy analogous positions on their surface as well. The possible tertiary structures of the three giardial proteins were determined using protein threading. While most positions on the modeled structures of the giardial SNAPs were occupied by residues analogous to those of the bovine $\alpha$-SNAP, there were few exceptions: (i) in GL50803_17224, a histidine (H158) was present in place of a canonical arginine or lysine; (ii) there was an interchange of position between an aromatic (F198) and a positively charged residues (K194) in GL50803_16521; and (iii) two positively charged residues (K159 and R160) were shifted more towards the edge of the concave face in GL50803_10856 (dotted circles in Fig. 1b; Additional file 2: Table S3). Barring these minor variations, the pattern of distribution of the positively-charged residues was mostly conserved for all the three giardial proteins and Sec17, indicating that they are likely to be functionally analogous.

Existing literature documents that two of the three $\alpha$-SNAP proteins, $\alpha-\mathrm{SNAP}_{17224}$ and $\alpha$-SNAP ${ }_{16521}$, are expressed in trophozoites and up to $14 \mathrm{~h}$ of encystation [30]. Reverse transcription PCR indicates that all the three identified genes are transcribed in trophozoites, encysting trophozoites (8 and $16 \mathrm{~h}$ following induction of encystation), and cysts (Additional file 1: Figure S2). Thus, the identified ORFs are not pseudogenes and the activity encoded by each is required in all stages of this parasite's life-cycle. Perusal of the genomes of the other Giardia isolates included in the GiardiaDB (assemblage A2 isolate $\mathrm{DH}$, assemblage B isolate GS, assemblage B isolate GS_B and assemblage E isolate P15) indicates that each of these also encodes three putative $\alpha$-SNAPs (data not shown). The presence of multiple $\alpha$-SNAP paralogues in the genome of a unicellular protist that lacks diversity of endomembrane compartments is unusual given that most eukaryotic genomes encode only one orthologue of this protein; exceptions include Arabidopsis thaliana, which has two (Additional file 2: Tables S1 and S2) [31]. To determine if the identified sequences are $\alpha$ - or $\gamma$-SNAPs, we reconstructed a phylogenetic tree with known $\alpha$ - and $\gamma$-SNAP sequences from diverse taxonomic groups. Sequences of putative SNAP paralogues from the closely-related diplomonad, S. salmonicida and Trepomonas sp., were also included. From the tree topology, we observed that while two sets of sequences, one with GL50803_17224 and another with GL50803_16521 of the reference strain (assemblage A, isolate WB), cluster together with the $\alpha$-SNAPs, the third set, with GL50803_10856, clusters closer to the $\gamma$-SNAPs (Fig. 2). Trepomonas, a close relative of Giardia, also contains three SNAP paralogues, of which two cluster with the $\gamma$-SNAPs and the third is within the $\alpha$-SNAP cluster. Unlike Giardia and Trepomonas, S. salmonicida encodes only two putative SNAPs, of which one clusters with the $\alpha$-SNAPs while the other with the $\gamma$-SNAPs. Based on this analysis, it appears that while two of the identified giardial sequences are likely to be $\alpha$-SNAPs, the third may be a $\gamma$-SNAP.

It is known that $\alpha$-, but not $\beta$ - or $\gamma$-SNAP can substitute for Sec17 [18]. We used this criterion to evaluate if the identified giardial proteins are $\alpha$ - or $\gamma$-SNAP orthologues. Towards this, we used a mutant yeast strain having the temperature-sensitive sec17-1 allele, which is functional at $30{ }^{\circ} \mathrm{C}$ but not at $37^{\circ} \mathrm{C}$ [32]. We used functional complementation to assess if any of three giardial genes can functionally substitute for the sec17-1 hypomorph. All three giardial genes were expressed in the sec17-1 mutant under the control of a constitutive promoter. Expression of yeast's own SEC17 gene from the same vector served as a positive control while yeast transformants containing just the vector backbone served as negative control (Fig. 3). All the five transformants exhibited equivalent growth at the permissive temperature of $30{ }^{\circ} \mathrm{C}$. At the non-permissive temperature of $37{ }^{\circ} \mathrm{C}$, 


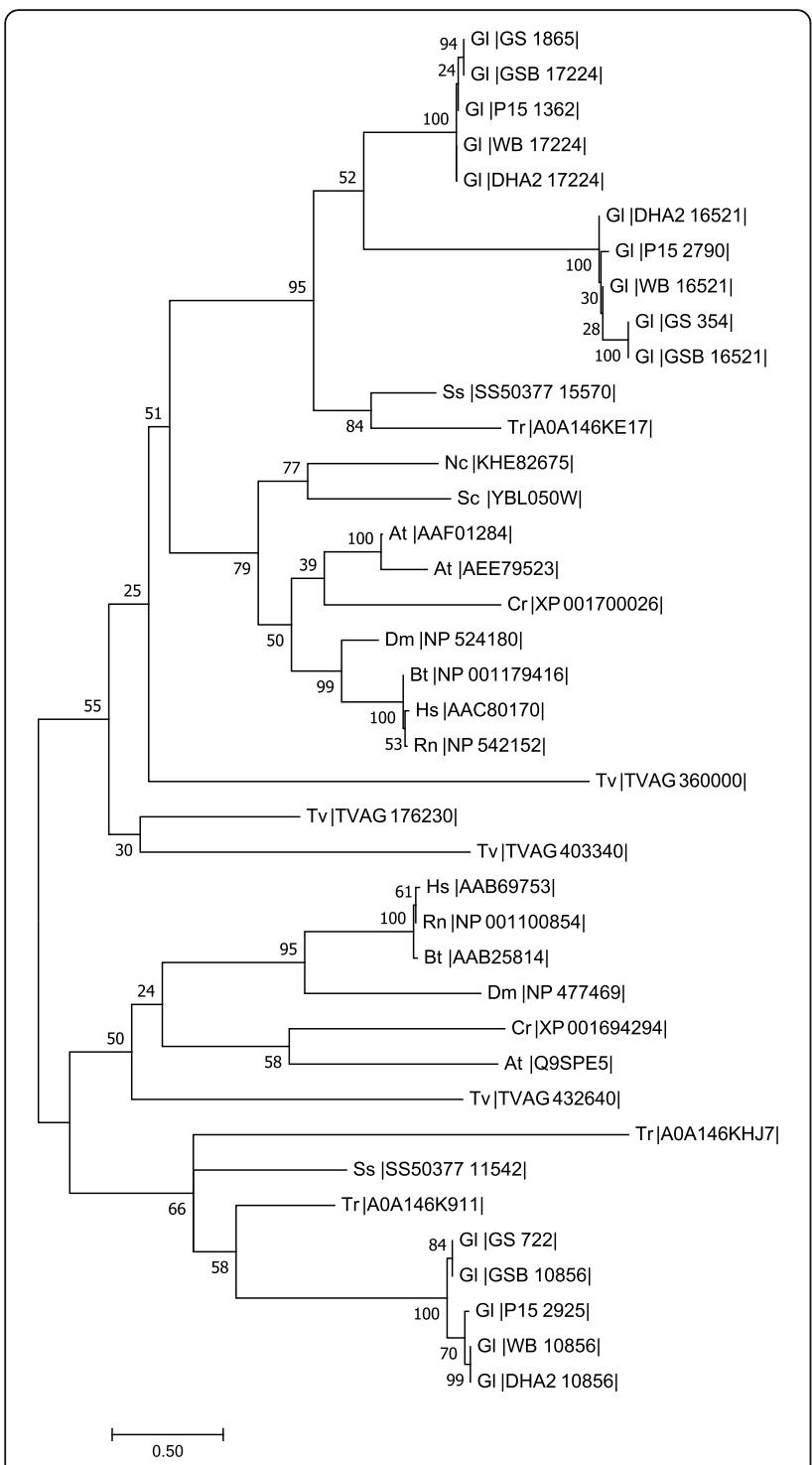

Fig. 2 Phylogenetic analysis of the putative SNAPs of G. lamblia. Sequences of $a-$ and $\gamma$-SNAPs from diverse taxonomic groups, along with those from all five isolates of $\mathrm{G}$. lamblia [assemblage $\mathrm{A}$ isolate WB (WB), assemblage A2 isolate DHA2 (DHA2), assemblage $E$ isolate P15 (P15), assemblage B isolate GS (GS) and assemblage B isolate GS_B (GSB)] were used to reconstruct a phylogenetic tree using the Maximum Likelihood algorithm. The accession numbers for all homologs used in the analysis are listed next to the organism's name, while the numerical value next to each node of the phylogenetic tree indicates bootstrap values obtained from 100 replicates. The names of the organisms have been abbreviated as follows: Gl, Giardia lamblia; Tr, Trepomonas sp.; Tv, Trichomonas vaginalis; Ss, Spironucleus salmonicida; Nc, Neurospora crassa; Sc, Saccharomyces cerevisiae; At, Arabidopsis thaliana; $\mathrm{Cr}$,

Chlamydomonas reinhardtii; Dm, Drosophila melanogaster; Bt, Bos taurus; Hs, Homo sapiens; Rn, Rattus norvegicus. The scale-bar represents the number of amino acid substitution for each site mutants expressing the plasmid-borne copy of SEC17 exhibited robust growth while those transformants harboring just the vector backbone failed to grow at this non-permissive temperature (Fig. 3). Growth of yeast cells expressing any one of the three giardial genes was comparable to the positive control. Thus, the survival of the temperature sensitive sec17-1 mutant expressing any of the three giardial genes at $37{ }^{\circ} \mathrm{C}$ indicates that even though one of the three SNAP sequences from Giardia clusters with $\gamma$-SNAPs, all these genes encode proteins that are functionally equivalent to Sec17 of yeast, which is an $\alpha$-SNAP. Based on the results of this complementation analysis, we conclude that all the three SNAP proteins of Giardia are orthologous to $\alpha$ - and not $\gamma$-SNAP.

\section{Unique subcellular localization of the three a-SNAPs indicate functional divergence}

The presence of three putative $\alpha$-SNAPs that are expressed in multiple stages of the parasite's life-cycle raises the possibility that these may have undergone functional divergence during the course of evolution. Thus, while one of them may be under strong selection pressure to discharge the essential functions of an $\alpha$-SNAP, the other(s) may have been adapted to perform alternative functions. To investigate if such functional divergence has taken place, we wanted to determine the subcellular distribution of the three $\alpha$-SNAPs. Towards this, we raised polyclonal antibodies against $\alpha-\mathrm{SNAP}_{17224}$ and $\alpha-\mathrm{SNAP}_{16521}$ in rabbit and against $\alpha-\mathrm{SNAP}_{10856}$ in mouse. Each of the polyclonal antibodies specifically detected only the corresponding protein that had been purified from $E$. coli as a band of $\sim 34 \mathrm{kDa}$; none of them detected any of the other two $\alpha$-SNAPs (Additional file 1: Figure S3a). Each antibody also detected a single band in G. lamblia trophozoite extract whose size corresponded to that predicted for the $\alpha$-SNAPs of Giardia (Additional file 1: Figure S3a). Thus, in the absence of any observed cross-reactivity, it can be concluded that each antibody recognizes its target $\alpha$-SNAP with a high degree of specificity. This also indicates substantial structural differences amongst these three proteins.

The polyclonal antibodies were used for immunolocalization of the three $\alpha$-SNAPs in all the different stages of the parasite life-cycle in which their expression had been detected previously (Additional file 1: Figure S2). Given that all three genes can functionally complement the sec17-1 temperature-sensitive allele (Fig. 3), it is expected that these proteins will be associated with vesicles and/or membrane compartments such as the ER. Consistently, a previous study has already reported the localization of $\alpha$-SNAP 16521 to the PVs [33]. We observed that along with $\alpha$-SNAP ${ }_{16521}, \alpha-\mathrm{SNAP}_{10856}$ also localizes to the PVs as the signal for this protein colocalizes with that of the fluorescent dye Lucifer yellow, which is endocytosed and delivered to acidic compartments (Fig. 4a; Additional file 


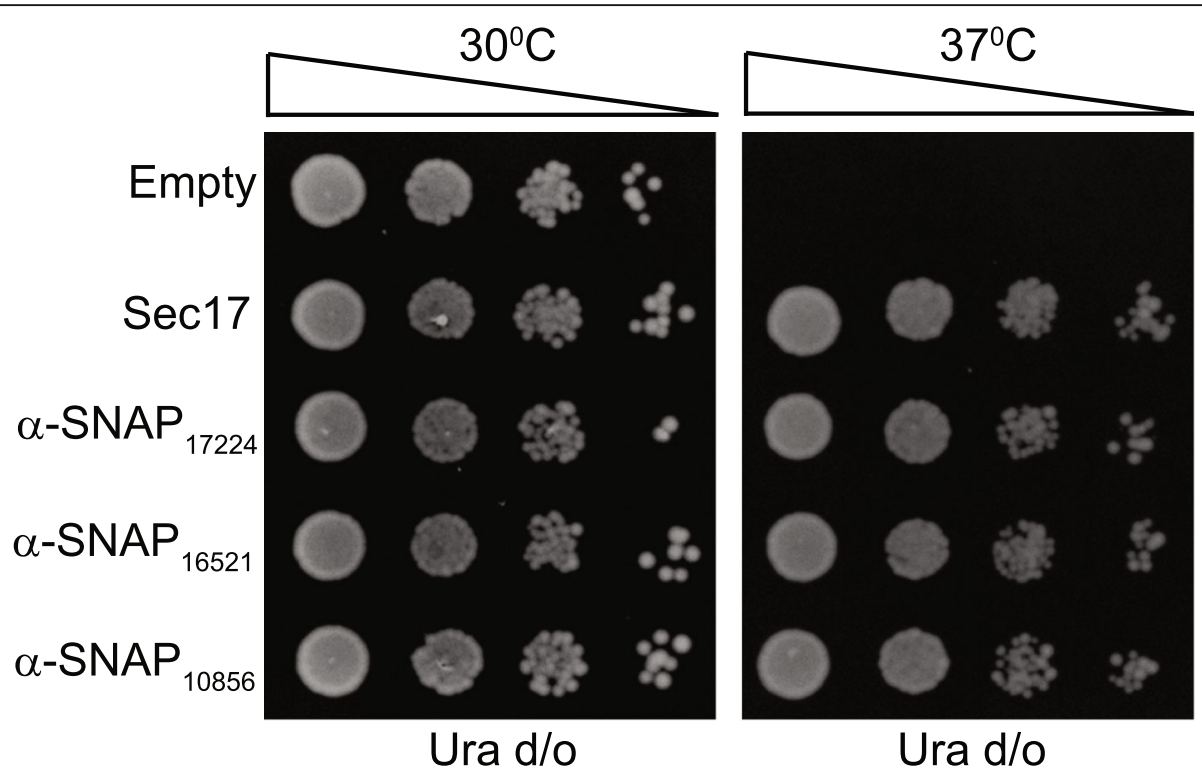

Fig. 3 Functional complementation of a temperature-sensitive a-SNAP mutant of yeast with the putative SNAPs of Giardia. Temperature-sensitive sec17-1mutant yeast strain (RSY269) was transformed with constructs expressing either SEC17 (positive control) or each of the giardial SNAPs; transformants carrying the empty vector served as negative control. Transformants were spotted onto synthetic medium lacking uracil and incubated at either $30^{\circ} \mathrm{C}$ (permissive temperature) or $37^{\circ} \mathrm{C}$ (non-permissive temperature)

1: Figure S4a). $\alpha$-SNAP 10856 also colocalizes with the PX domain-containing protein encoded by the ORF GL50803_16548, which is known to localize to the PVs (Additional file 1: Figure S4b) [34, 35]. Unlike the other two, $\alpha-\mathrm{SNAP}_{17224}$ localized to the anterior part of the cell, mostly around the two nuclei; another pool of the protein appeared to be cytoplasmic (Fig. 4a). This difference in the subcellular localization between $\alpha-\mathrm{SNAP}_{17224}$ and the two other $\alpha$-SNAP paralogues indicates that the former performs a specific function that is different from that of the other two. Thus, there appears to be a functional divergence for at least one of the three $\alpha$-SNAPs.

We observed additional evidence of such functional divergence in trophozoites undergoing encystation. While the pattern of localization of $\alpha-\mathrm{SNAP}_{10856}$ in trophozoites and encysting cells remained the same, that of the other two paralogues changed significantly (Fig. 4a). At 8 and $16 \mathrm{~h}$ after induction of encystation, $\alpha$-SNAP ${ }_{17224}$ still localized at the perinuclear regions, but its cytoplasmic distribution was no longer evident. Such a distribution persisted even after $30 \mathrm{~h}$ of induction (Additional file 1: Figure S5a). However, at $48 \mathrm{~h}$ post-induction, it was exclusively located at the PVs, with no signal at the perinuclear region (Fig. 4a; Additional file 3: Figure S9 and Additional file 2: Table S6). $\alpha$-SNAP ${ }_{16521}$ also underwent a change in cellular distribution during encystation, but this change was much more rapid compared to $\alpha$-SNAP ${ }_{17224}$. At 8 and $16 \mathrm{~h}$ post-induction, $\alpha-\mathrm{SNAP}_{16521}$ was present exclusively at the paraflagellar dense rods (PDRs), which are electron-dense structures that are associated with the anterior, caudal and posteriolateral flagella (Fig. 4a; Additional file 1: Figure S5b and c, Additional file 3: Figure S9 and Additional file 2: Table S6) [36]. This transition from the PVs to the PDRs starts as early as $1.5 \mathrm{~h}$ after the start of encystation as such cells exhibit both peripheral as well as distribution to the PDRs of the anterior and caudal flagella; complete redistribution of the signal appears to be completed by $4 \mathrm{~h}$ (Additional file 1: Figure $\mathrm{S} 5 \mathrm{~b})$. The signal was more prominent at the PDRs of the anterior flagella, compared to that of the posteriolateral or caudal flagella. At the $48 \mathrm{~h}$ time-point, besides the signal at the PDRs, $\alpha$-SNAP 16521 was also observed at the periphery of the ventral disc (Fig. 4a and Additional file 1: Figure S5d). This $\alpha$-SNAP continued to associate with flagellar structures even in the tetranucleated cysts and also localized to cytoplasmic puncta (Fig. 4a). $\alpha-\mathrm{SNAP}_{17224}$ and $\alpha-\mathrm{SNAP}_{10856}$ also exhibited a similar punctate distribution in cysts, without any association with the flagella. These puncta are likely to be vesicles as many of these are also positive for the lipid-binding PX domain-containing protein mentioned above (Additional file 1: Figure S6). Although the change in the pattern of subcellular distribution during encystation is unique to each $\alpha$-SNAP, the pattern of expression of the corresponding genes was very similar during this period. Real-time PCR indicated that the expression of all the three genes remained largely unchanged during encystation, with significant downregulation observed only in cysts (Fig. 4b, lower panel). The expression of the gene 

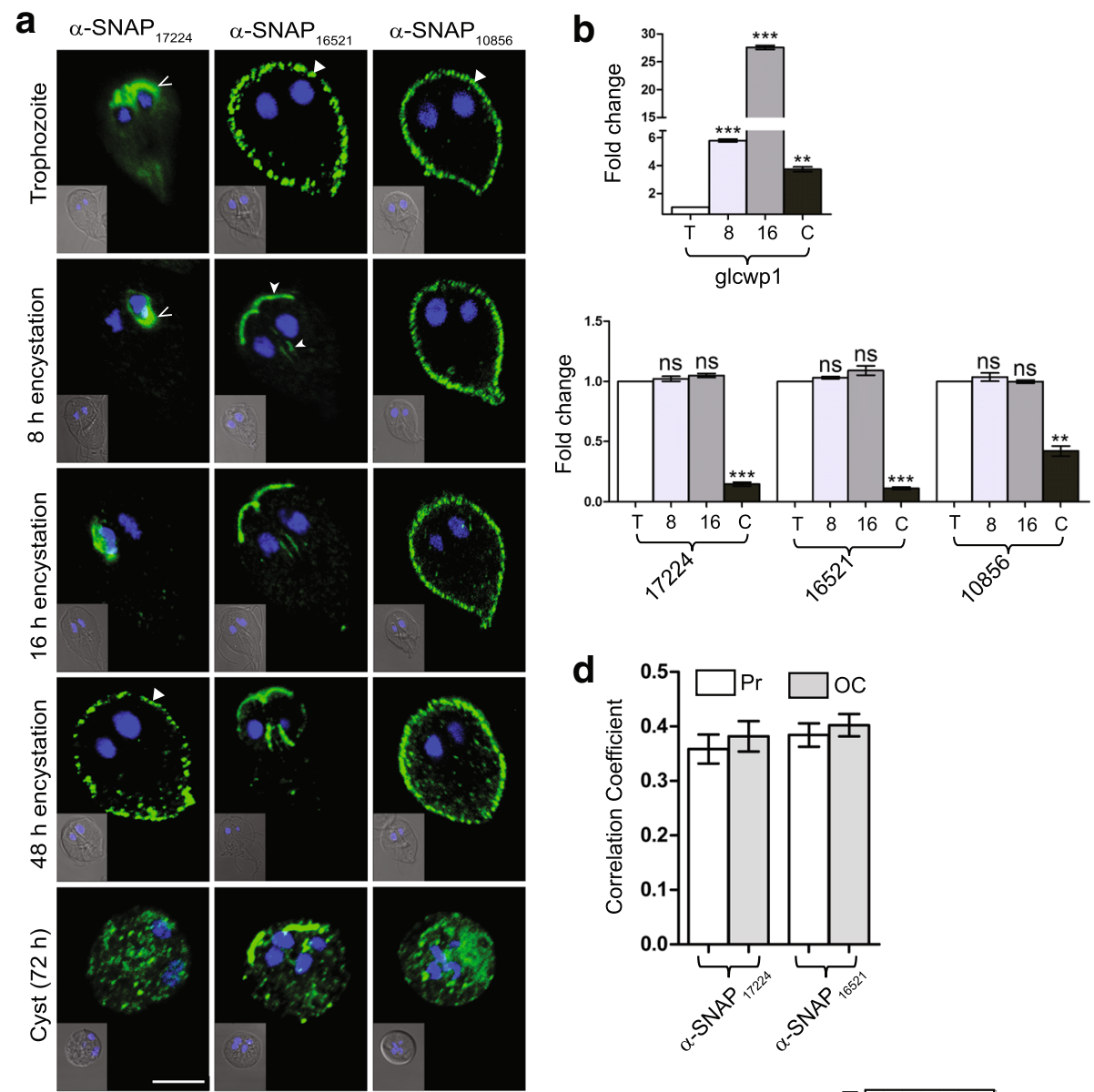

C
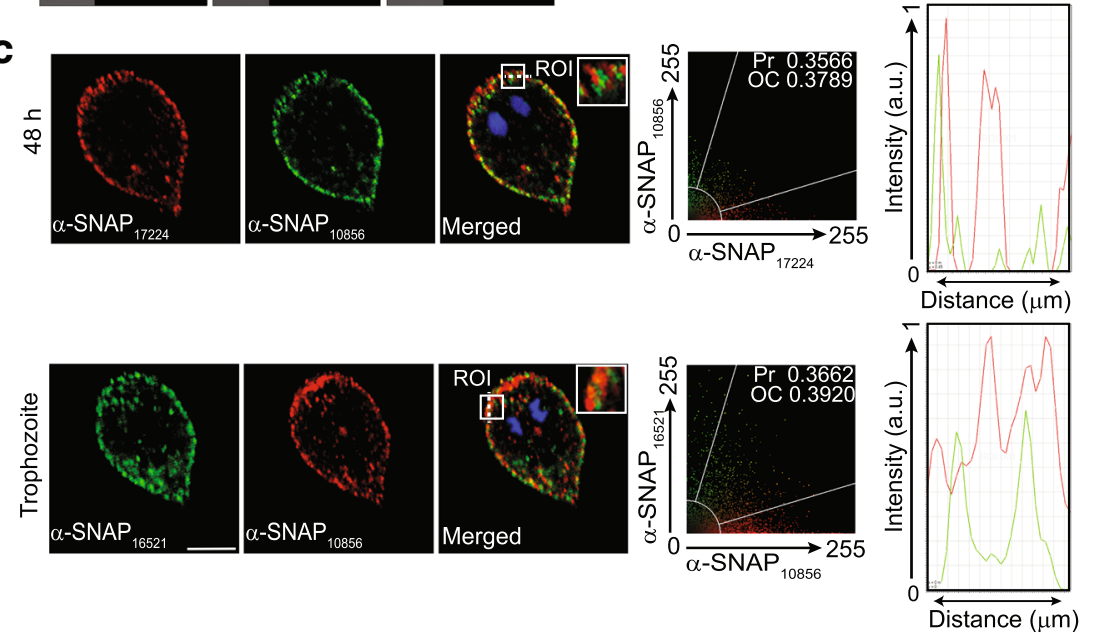

Fig. 4 (See legend on next page.) 


\begin{abstract}
(See figure on previous page.)
Fig. 4 Localization and expression of Giardia a-SNAPs in trophozoites, encysting trophozoites and cysts. a Immunofluorescence localization, with polyclonal antibodies, of a-SNAP 17224 (left column), a-SNAP 16521 (middle column) and a-SNAP 10856 (right column) in trophozoites, encysting trophozoites $(8,16$ and $48 \mathrm{~h}$ post-induction of encystation) and cysts. The caret marks the perinuclear region, the arrowhead marks the PDR, and the triangle indicates PVs. To show the localization of a-SNAP 16521 at both the PDRs and the ventral disc periphery (48 h post-induction), the corresponding panel is an overlay of two z-sections (individual images of the z-stack shown in Additional file 1: Figure S5d). Inset depicts overlay of the DIC and DAPI images. $\mathbf{b}$ Expression of the a-SNAP genes in trophozoites, encysting trophozoites and cysts were determined by real-time PCR (lower panel), where the expression of CWP1 gene serves as a positive control (upper panel). The asterisks indicate the significance of the difference between the expression under a given condition with that in trophozoites ( ${ }^{* *} P<0.01$; ${ }^{* *} P<0.001$; ns, not significant). c Colocalization of a-SNAP 17224 and a-SNAP 10856 in $48 \mathrm{~h}$ encysting trophozoites (top row) or that of a-SNAP 16521 and a-SNAP ${ }_{10856}$ in trophozoites (bottom row). Insets depict magnification of the region of interest (ROI) that has been marked with a white box. The scattergram in each row indicates the analysis of colocalization between the two fluorophores over the entire z-stack by considering all the pixels within the whole area occupied by that cell. The values for Pearson correlation coefficient (Pr) and overlap coefficient (OC) written inside the scattergrams. The intensity plots at the extreme right indicate changes in fluorescence intensity of the red and green signals across the dotted white line in the ROI. $\mathbf{d}$ Mean Pr and OC values for multiple z-stacks to determine the extent of colocalization of either a-SNAP ${ }_{17224}$ or a-SNAP ${ }_{16521}$ with a-SNAP ${ }_{10856}$. Scale-bars: a, c, $5 \mu$ m
\end{abstract}

encoding CWP1 served as a positive control (Fig. 4b, upper panel) [37]. Such subcellular redistribution of proteins, without any change at the level of transcription, has been previously observed for other giardial proteins, such as $\beta$ 'COP subunit, Rab11, YiP, heavy chain of clathrin, DRP, ESCP and Rpn10 [13, 38-40]. Thus, many regulatory changes in this protist appear to be dependent on protein relocation, rather than synthesis of new proteins.

There are two situations in which two of the $\alpha$-SNAPs exhibit a similar peripheral distribution in trophozoites or encysting trophozoites: (i) $\alpha-\mathrm{SNAP}_{16521}$ and $\alpha-\mathrm{SNAP}_{10856}$ in trophozoites and (ii) $\alpha-\mathrm{SNAP}_{17224}$ and $\alpha-\mathrm{SNAP}_{10856}, 48$ $h$ post-induction of encystation (Fig. 4a). To determine if the two above-mentioned protein pairs colocalize, we carried out quantitative colocalization analysis for each pair under the conditions in which they exhibit similar cellular distribution. Scattergrams of multiple images of both the protein pairs did not indicate any substantial colocalization as the distributions of the green and red-colored pixels did not overlap significantly (Fig. 4c). Both the scattergram and the intensity plot of the two different fluorophores indicate that while there was some overlap for $\alpha-\mathrm{SNAP}_{16521}$ and $\alpha-\mathrm{SNAP}_{10856}$ in trophozoites, the same was not observed for $\alpha-\mathrm{SNAP}_{17224}$ and $\alpha-\mathrm{SNAP}_{10856}$, in encysting trophozoites (Fig. 4c). This is supported by colocalization analysis of multiple images wherein both the Pearson's correlation (Pr) and overlap coefficient (OC) values are below 0.5 for each protein pair (Fig. 4d). Incidentally, SNAP 16521 and $\alpha$-SNAP ${ }_{17224}$ never localize to the same subcellular region under any of the conditions tested (trophozoites, encysting trophozoites and cysts) (Fig. 4a). Given this lack of colocalization of the three $\alpha$-SNAP paralogues, it may be concluded that there are functional differences among these three proteins.

\section{All the a-SNAP paralogues colocalize with NSF}

Even though all the three paralogues function as $\alpha$-SNAP in yeast, they exhibit heterogeneity in terms of their localization in Giardia. This scenario may arise if one or more of these paralogues do not function as an $\alpha$-SNAP. Since $\alpha$-SNAP and NSF assemble into the functional $20 \mathrm{~S}$ complex that brings about SNARE unwinding, we examined if the three paralogues colocalize with the giardial NSF (GINSF), which is encoded by the ORF 50803_114776. Immunolocalization of GINSF in trophozoites was performed with an antibody (raised in rat) that specifically recognized this protein in giardial protein extract (Additional file 1: Figure S3b). It was observed that GINSF localized to the PVs and the "brush border" structures associated with the part of the anterior flagella that is proximal to the basal bodies (Fig. 5a) [41]. This distribution persisted in $48 \mathrm{~h}$ encysting cells, with the enhanced signal at the brush borders. While none of the $\alpha$-SNAPs localized to the brush borders, all of them exhibit a peripheral distribution in either trophozoites or encysting trophozoites (Fig. 4a). Hence for colocalization of the $\alpha$-SNAPs with GINSF, conditions were chosen where each of the $\alpha$-SNAPs exhibit a predominantly peripheral distribution, $48 \mathrm{~h}$ encysting trophozoites for $\alpha-\mathrm{SNAP}_{17224}$, trophozoites for $\alpha$-SNAP 16521 , and both trophozoites and encysting trophozoites $(48 \mathrm{~h})$ for $\alpha-\mathrm{SNAP}_{10856}$ (Fig. 5a). Under each of the conditions tested, all the three $\alpha$-SNAPs showed significant colocalization with the GINSF at the cell periphery, but not at the brush borders of the anterior flagella (Fig. 5a, Additional file 1: Figure S7). Both the Pr and the OC values indicate significant colocalization in multiple cells (Fig. 5b). Since all three $\alpha$-SNAPs colocalize with GINSF and each of them functionally complements the sec17-1 temperature-sensitive allele (Fig. 3), it is likely that they function as $\alpha$-SNAP in Giardia. Interestingly, barring $\alpha-\mathrm{SNAP}_{10856}$, the two other $\alpha$-SNAPs and GINSF localize to regions where there is no overlap of the SNAP and NSF signals. These include the perinuclear regions for $\alpha-\mathrm{SNAP}_{17224}$, the PDR for $\alpha-\mathrm{SNAP}_{16521}$, and the brush borders for GINSF. Thus, it seems likely that in addition to functioning in the $20 \mathrm{~S}$ complex, each of these proteins also discharges additional cellular function(s). 

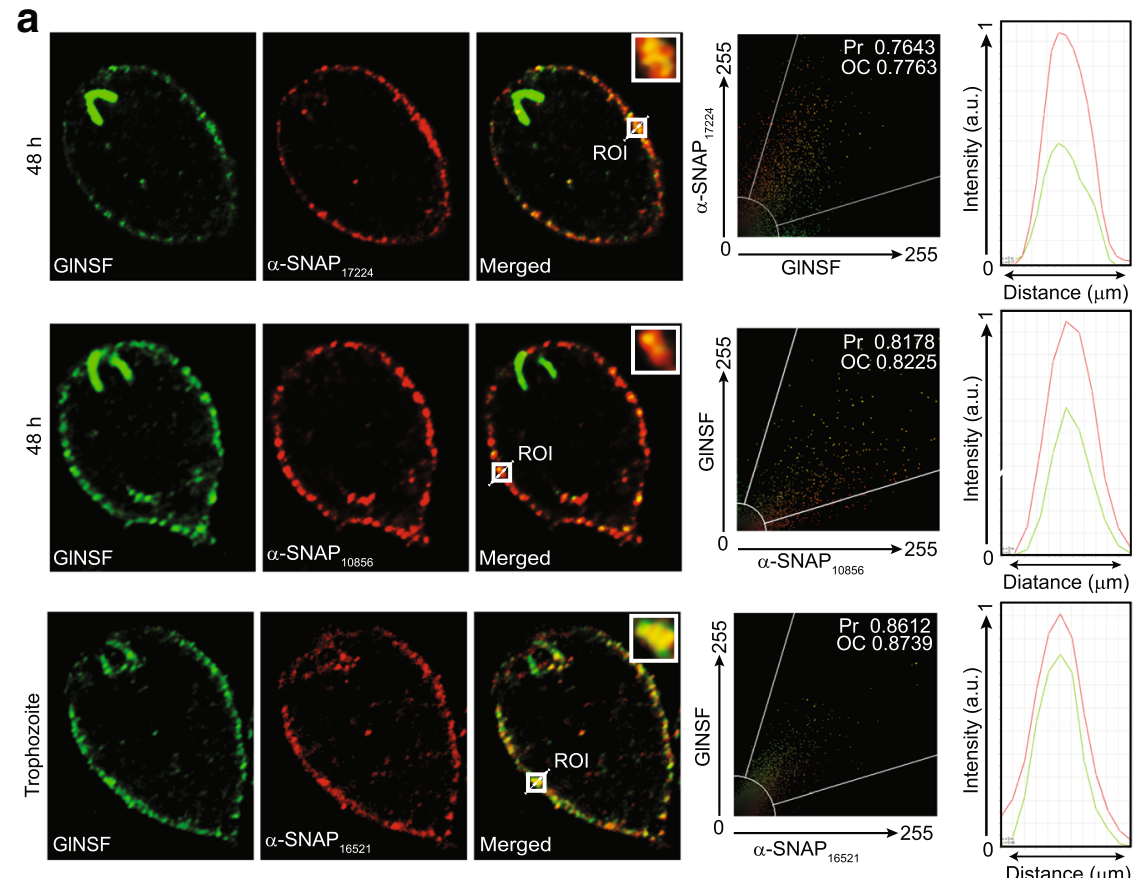

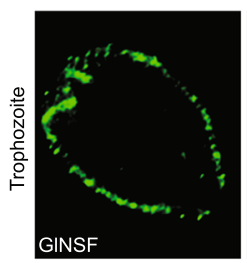

b
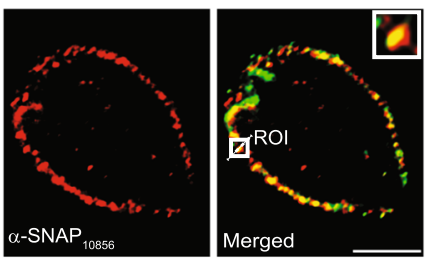

C

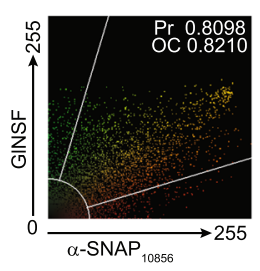

d
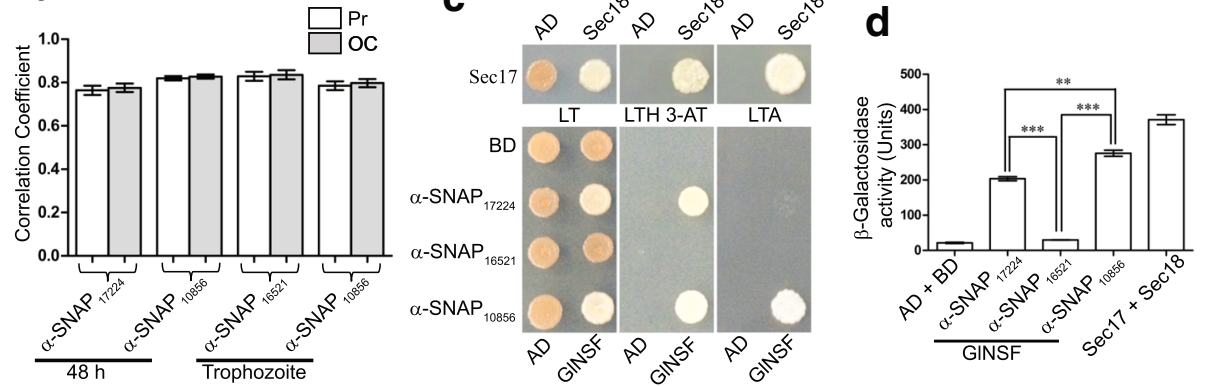

Fig. 5 Colocalization and binary interaction between GINSF and the giardial a-SNAPs. a Colocalization of GINSF with a-SNAP 17224 Or a-SNAP 10856 in $48 \mathrm{~h}$ encysting trophozoites, and with a-SNAP ${ }_{16521}$ or a-SNAP ${ }_{10856}$ in trophozoites. Insets depict magnification of the ROI (marked with a white box). The scattergram in each row indicates the analysis of colocalization between the two fluorophores over the entire $z$-stack by considering the pixels within the entire area occupied by the particular cell. The values for Pearson correlation coefficient (Pr) and overlap coefficient (OC) are indicated inside the scattergrams. The intensity plots at the extreme right indicate changes in the intensity of the red and green fluorescence signals across the diagonal of the ROI depicted by a dotted white line. $\mathbf{b}$ The bar graph denotes the mean Pr and OC calculated from the $z$-stacks of six independent images. c PJ69-4A cells were transformed with various combinations of constructs expressing fusion proteins with either Gal4 DNA binding domain (BD) or its activation domain (AD). Expression of the BD or AD alone served as negative controls. Transformants were spotted on YCM plates lacking leucine and tryptophan (LT), or leucine, tryptophan and histidine with $2.5 \mathrm{mM}$ 3-AT (LTH 3-AT), or leucine, tryptophan and adenine (LTA). d $\beta$-galactosidase activity of the indicate transformants was quantified. Statistical significance of the difference in interaction between any two interacting pairs is indicated $\left.{ }^{* *} P<0.01,{ }^{* *} P<0.001\right)$. Scale-bar: $\mathbf{a}, 5 \mu \mathrm{m}$

Difference in the interactions between GINSF and each of the three a-SNAPs

Given that the three $\alpha$-SNAP paralogues colocalize with GINSF, we wanted to test whether each of these three proteins is capable of directly interacting with the latter. We used yeast two-hybrid to assess this binary interaction (Fig. 5c). It is known that yeast Sec17 physically interacts with its own NSF (Sec18) [18]. Consequently, 
these two proteins exhibit strong interaction in our two-hybrid assay as cells co-expressing Sec17 from the bait vector (pGBT9 with TRP1 selection marker) and Sec18 from the prey vector (pGAD424 with LEU2 selection marker), exhibited robust growth, not only on plates lacking histidine (LTH 3-AT), but also on plates lacking adenine (LTA); in comparison to the former, the later selection medium imposes greater stringency as only cells harboring strongly interacting bait-prey pairs can grow in the absence of exogenously-added adenine [42]. Results of the spot assay indicated that the interaction between GINSF and $\alpha-\mathrm{SNAP}_{10856}$ was comparable to that between Sec17 and Sec18 as yeast transformants expressing this giardial protein pair were able to grow on both LTH 3-AT and LTA plates (Fig 5c). However, estimation of the activity of the $L a c Z$ reporter gene, which provides a quantitative estimation of binary interaction, indicates that the interaction between the giardial proteins was weaker compared to that between the yeast proteins (Fig. 5d). Transformants expressing GINSF and $\alpha-\mathrm{SNAP}_{17224}$ exhibited growth on LTH 3-AT, but not on LTA plates (Fig. 5c). Even the $\beta$-galactosidase activity indicates that this giardial $\alpha$-SNAP's interaction with GINSF was weaker compared to that between GINSF and $\alpha-\mathrm{SNAP}_{10856}$ (Fig. $5 \mathrm{~d}$ ). However, there does not appear to be any interaction between GINSF and $\alpha-\mathrm{SNAP}_{16521}$ as there is no growth on both LTA and LTH 3-AT plates. Even the color of the spot growing on plates lacking leucine and tryptophan (LT) is comparable to those of the negative control (Fig. 5c), as is the $\beta$-galactosidase activity of this transformant (Fig. $5 \mathrm{~d}$ ). Based on these observations there appears to be a lack of binary interaction between $\alpha-\mathrm{SNAP}_{16521}$ and GINSF; however, we cannot rule out an interaction between these two proteins in vivo where additional cellular factors may stabilize this interacting pair. Taken together, the results of the yeast two-hybrid assay indicate that GINSF interacts differentially with the three $\alpha$-SNAPs, with the strongest interaction taking place with $\alpha-\mathrm{SNAP}_{10856}$, followed by that with $\alpha-\mathrm{SNAP}_{17224}$, and very little or no interaction with $\alpha-\mathrm{SNAP}_{16521}$. This observed difference in the interaction of the three paralogous proteins with GINSF further underscores the fact that the three giardial $\alpha$-SNAPs are likely to have undergone functional divergence in this protist.

\section{Oxidative stress induced relocalization of a-SNAPs}

Based on the observed relocalization of $\alpha-\mathrm{SNAP}_{17224}$ and $\alpha-\mathrm{SNAP}_{16521}$ during encystation, we hypothesize that change in subcellular distribution of these two $\alpha$-SNAPs may be part of Giardia's response to changes in the external environment. If this hypothesis is true, then similar changes may occur when cells encounter oxidative stress. We chose oxidative stress in particular because existing literature indicates that Giardia is likely to have a unique mechanism for handling this stress. Not only is Giardia unable to tolerate elevated oxygen levels, it lacks several key components that are used by most eukaryotes to detoxify cellular reactive oxygen species [43]. Thus, while Giardia lacks enzymes such as catalase, glutathione peroxidase, and superoxide dismutase, its defense against oxidative stress includes noncanonical enzymes like an $\mathrm{NADH}$ oxidase, an NADH peroxidase, and a flavodiiron protein, to name a few [44]. To investigate if the presence of multiple paralogues of $\alpha$-SNAP may be a part of this organism's atypical mechanism to combat oxidative stress, we monitored the cellular distribution of these three proteins after inducing oxidative stress in trophozoites with two different agents, $\mathrm{H}_{2} \mathrm{O}_{2}(150 \mu \mathrm{M})$ and metronidazole $(1 \mu \mathrm{g} / \mathrm{ml})$ $[45,46]$. Treatment with both reagents for a period of $1 \mathrm{~h}$ was sufficient to induce intracellular oxidative stress, as monitored by the conversion of DCFDA to the fluorescent DCF (Additional file 1: Figure S8). Following induction of oxidative stress, the relocalization of $\alpha-\mathrm{SNAP}_{17224}$ and $\alpha-\mathrm{SNAP}_{16521}$ was similar to that previously observed during the late stages of encystation (Compare Fig. $4 \mathrm{a}$ and Fig. 6; Additional file 3: Figure S10a and b, Additional file 2: Table S7). Thus, while $\alpha-\mathrm{SNAP}_{16521}$ relocalized from the cell periphery to the PDRs, the signal for $\alpha-\mathrm{SNAP}_{17224}$ moved from the perinuclear region to the cell periphery.
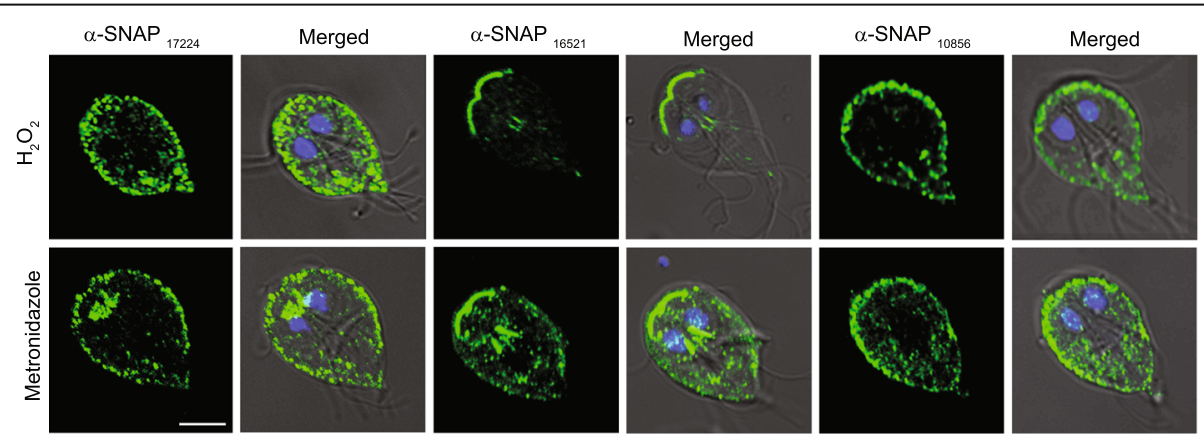

Fig. 6 Localization of giardial a-SNAPs following oxidative stress. Localization of the three a-SNAPs in trophozoites exposed for $1 \mathrm{~h}$ to either 150 $\mu \mathrm{m} \mathrm{H}_{2} \mathrm{O}_{2}$ (top row) or $1 \mu \mathrm{g} / \mathrm{ml}$ metronidazole (bottom row). Scale-bar: $5 \mu \mathrm{m}$ 
However, unlike the change observed during encystation, a punctate cytoplasmic signal was also observed in both cases, with a cluster of puncta at the perinuclear region especially in case of $\alpha-\mathrm{SNAP}_{17224}$ (Fig. 6). Consistent with the observed lack of change in subcellular distribution during encystation (Fig. 4a), $\alpha$-SNAP ${ }_{10856}$ remained at the cell periphery even during oxidative stress (Fig. 6). However, like the other two $\alpha$-SNAPs, cytoplasmic puncta were also observed in this case. Thus, in contrast to $\alpha-\mathrm{SNAP}_{10856}$, the change in the distribution of $\alpha-\mathrm{SNAP}_{17224}$ and $\alpha-\mathrm{SNAP}_{16521}$ during both encystation and oxidative stress indicates that these two paralogues may have acquired additional functions during the course of evolution.

\section{Discussion}

Although most eukaryotes encode only one $\alpha$-SNAP, here we report that there are three paralogues in Giardia. These three genes are expressed in multiple morphological states of the parasite, thereby indicating that these three proteins are unlikely to discharge redundant functions in these different stages. Although $\alpha-\mathrm{SNAP}_{10856}$ clusters with $\gamma$-SNAP sequences in the reconstructed phylogenetic tree, the results of the complementation analysis clearly indicate that it is functionally orthologous to $\alpha$-SNAP (Fig. 3). The presence of three functional $\alpha$-SNAP paralogues in a unicellular organism appears to be in stark contrast to most multicellular eukaryotes having only one homolog. Interestingly, the presence of multiple SNAPs has been documented in 47 protist genomes, including those of Trichomonas, Entamoeba, Naegleria, Trypanosoma and Leishmania and in silico analyses indicate that some of these putative proteins may be $\gamma$-SNAPs [47] (Dirk Fasshauer, personal communication). We have identified two SNAP paralogues in S. salmonicida and three in Trepomonas (Fig. 2). Thus, the presence of multiple SNAPs seems to be the norm for protists. Given that Protista represents a paraphyletic group, it is not possible to ascertain if a single gene duplication event gave rise to the expansion of the SNAP family, or if there were multiple independent such events. However, the fact that there is a difference in the number of SNAPs in closely-related diplomonad species, two in S. salmonicida and three in both Giardia and Trepomonas, indicates that later duplication events have also contributed to the expansion of this family of proteins in Protista.

While the results of the complementation assay indicate that each of the three paralogues are functionally analogous to Sec17 of yeast, the distinct subcellular localization of each protein in Giardia indicates that they do not perform redundant functions. In addition, results of the two-hybrid assay indicate that GINSF had varying affinity for the three $\alpha$-SNAPs. Given that GINSF binds to $\alpha$-SNAP ${ }_{10856}$ with the highest affinity, it may be hypothesized that the bulk of giardial cis-SNARE unpairing may be carried out by this paralogue. Its localization to the PV, where most of the SNAREs are present, lends support to this hypothesis [48]. Notably, this distribution does not change even during encystation, and after exposure to oxidative stress. Conversely, GINSF has little or no interaction with $\alpha-\mathrm{SNAP}_{16521}$ and this paralogue relocalizes to PDR region where neither GINSF nor the SNAREs are documented to be present, suggesting that NSF attachment may not be necessary for the function(s) this protein discharges at the PDR. However, since $\alpha$-SNAP 16521 localizes to the PVs in trophozoites, its participation in SNARE complex disassembly cannot be ruled out.

One reason for the presence of such complexity in this unicellular protist may be the necessity to maintain Giardia's asymmetric cell morphology. Unlike amoeboid protists, Giardia has a very unique tear-drop shape. The maintenance of this distinct asymmetric shape is likely to entail polarized vesicular trafficking to selective regions of the cell surface. However, the morphologically-simple endomembrane system of Giardia appears to lack key sorting stations, such as the Golgi. In the absence of readily-identifiable sorting compartments, Giardia may have evolved another system of determinants that allow selective targeting of vesicles to specific regions of the plasma membrane so that the shape of the cell is maintained. $\alpha$-SNAP is already known to participate in such selective targeting in metazoans; it has been documented to enable polarized sorting to the apical surface of neuroepithelium as a single mutation causes missorting of apical proteins such as E-cadherin, $\beta$-catenin and F-actin [49]. The factors that play a role in giardial cell shape maintenance are difficult to trace as mutant hunts cannot be undertaken in this polyploid organism. Thus, it would be interesting to determine if there are any morphological changes following selective ablation of any one of these $\alpha$-SNAPs.

The presence of $\alpha-\mathrm{SNAP}_{17224}$ and $\alpha-\mathrm{SNAP}_{16521}$ at cellular locations where NSF is not detectable is intriguing (Figs. 4a, 5a). One possibility is that $\alpha$-SNAP may be performing NSF-independent functions at these locations. Existing literature indicates that $\alpha$-SNAP has various NSF-independent roles [50]. An example of this is $\alpha$-SNAP's involvement in regulating calcium uptake via calcium release activated calcium (CRAC) channels [22]. Hexameric assemblies of the Orail protein at the PM forms the CRAC channel pores and this assembly process is regulated by $\alpha$-SNAP as its deficiency results in loss of calcium selectivity of the CRAC channels, leading to dysregulated entry of sodium into the cell [51]. In another instance, $\alpha$-SNAP interacts directly with the Bcl-2 family member, BNIP1, which is known to regulate the morphology of the ER [52]. Incidentally, we have also observed enhanced $\alpha-\mathrm{SNAP}_{17224}$ signal at perinuclear regions, which are known to be occupied by the 
ER (Fig. 4a). The distribution of $\alpha-\mathrm{SNAP}_{16521}$ at the PDRs is also indicative of a noncanonical role for this paralogue. Other proteins of Giardia with similar localization at the PDR include four proteins belonging to the Nek family of kinases (ORFs 5375, 92498, 16279 and 101534) and the catalytic subunits of protein phosphatase 2A (PP2Ac) and protein kinase A (PKAc) [53-55]. Nek proteins are documented to regulate ciliary function and assembly [56]. They also coordinate ciliary function with the cell cycle as they regulate the function of the centrioles, which serve both as basal bodies and microtubule-organizing centers [57]. Consistently, a study encompassing a large number of unikont and bikont organisms indicated that there is a direct correlation between the number of Nek genes encoded by a given genome and the presence of diving ciliated cell(s) in that particular organism [58]. Giardia has 56 active Neks, although its genome encodes 198; no other genome encodes such a large number of Neks and it is hypothesized that the expansion of this family of kinases is necessary to solve the challenges of coordinating the division of eight flagella with the cell cycle $[58,59]$. Both PKAc and PP2Ac localize to the PDR in trophozoites [53, 54]. Since the signal for both proteins disappear simultaneously from the PDR of the anterior flagella early in encystation, it is postulated that they function in the same pathway in Giardia $[53,54]$. PKAc is a known regulator of flagellar motility through its phosphorylation of dynein [60]. Thus, it is possible that since several other proteins that regulate flagellar function also localize to the PDRs, $\alpha-\mathrm{SNAP}_{16521}$ may play a similar role. The difference in the $\alpha-$ SNAP $_{16521}$ signal intensity at various PDRs may be because of differences in motility of each flagellar pair [61]. It may be noted that we have not detected the $\alpha$-SNAPs at all the subcellular location where SNAREs of Giardia are known to localize [48]. For example, while gQb3 localizes to mitosomes, we have not observed similar distribution for any of the $\alpha$-SNAPs. However, we cannot rule out the presence of a minor, and therefore undetectable, pool of any of the three $\alpha$-SNAPs at other locations within the cell.

\section{Conclusions}

The present study has uncovered the presence of three paralogues of $\alpha$-SNAPs in G. lamblia. Expression of these three genes during multiple morphological states of the protist indicates that the function of each of these three $\alpha$-SNAPs is required by the cell. Thus, these must be performing non-redundant functions. Antibodies raised against each of these three proteins were used to determine their subcellular distribution during different stages of the parasite's life-cycle, and also upon exposure of the trophozoites to oxidative stress. These immunofluorescence experiments indicated a non-overlapping pattern of subcellular redistribution, without any accompanying change in the expression of the corresponding genes. In addition, two-hybrid assay established that these three paralogues have varying affinity for GINSF. Taken together, it may be concluded that the three $\alpha$-SNAP paralogues have undergone functional divergence in this protist. It was observed that $\alpha$-SNAP ${ }_{16521}$ localizes to the PDRs associated with the anterior, posterolateral and caudal flagellar pairs. Given that nither GINSF nor any of the SNAREs localize to the PDRs, it is possible that this paralogue performs an NSF-independent function. Thus, following duplication of the $\alpha$-SNAP genes of Giardia, there may have been neofunctionalization of some of the paralogues.

\section{Methods}

Sequence analyses and secondary structure predictions The protein sequences of Giardia SNAPs were curated from GiardiaDB and secondary structure predictions were carried out using iterative threading assembly refinement (I-TASSER) and Protein Homology/analogY Recognition Engine (Phyre2) servers [62, 63]. By default, both approaches used the crystal structure of $S$. cerevisiae Sec17 (PDB ID: 1QQE) as a template [27]. Based on these predictions, the secondary structural elements were marked on the multiple-sequence alignment that was generated with ClustalW, with editing in Jalview $[64,65]$. Three-dimensional automatic threading models were generated in I-TASSER server, with the crystal structure of Sec17 as a template. The conserved positively charged residues were marked in Pymol [66].

\section{Phylogenetic analysis}

Sequences of both $\alpha$ - and $\gamma$-SNAPs from organisms were curated using either NCBI (https://www.ncbi.nlm.nih.gov), UniProt (https://www.uniprot.org) or Eukaryotic Pathogen Database Resources (https://eupathdb.org) [67-69]. Domain analysis was performed with Pfam to ensure that the identified sequences contained a SNAP domain [70]. The curated sequences were used to reconstruct a maximum likelihood tree using MEGA7, with 100 bootstrap replicates [71].

\section{Giardia lamblia culture and in vitro encystation}

Trophozoites of Assemblage A isolate Portland-1 were grown in slanted $15 \mathrm{ml}$ culture tubes containing Diamond TYI-S-33 medium ( $\mathrm{pH}$ 6.8) and the encystation was carried out as previously described [72, 73]. In order to obtain a pure preparation of cysts, $72 \mathrm{~h}$ post-induction of encystation, cells were harvested and incubated in distilled water at $4{ }^{\circ} \mathrm{C}$, for $24 \mathrm{~h}$ for selective lysis of trophozoites that did not undergo encystation. 


\section{Functional complementation}

The temperature-sensitive strain RSY269 (MAT $\alpha$ ura3-52 his4-619 sec17-1) was used for functional complementation [32]. SEC17 and all the three Giardia $\alpha$-SNAPs were PCR amplified with primers are listed in Additional file 2: Table S4. The PCR products were cloned under the control of a constitutive yeast promoter, in a $2 \mu \mathrm{m}$ vector having $U R A 3$ as a selectable marker (Additional file 2: Table S5). Each construct was individually transformed into RSY269. Resulting transformants were grown overnight in liquid YCM lacking uracil; serial dilutions of these cultures were spotted onto YCM plates lacking uracil and incubated at 30 and $37^{\circ} \mathrm{C}$.

\section{Polyclonal antibodies against giardial a-SNAPs and NSF}

Each of the three giardial $\alpha$-SNAPs was expressed and purified from BL21 (DE3) as previously described, except $0.2 \mathrm{mM}$ IPTG was used [17]. The N-terminal region of GINSF was also induced with the same concentration of IPTG but was purified from the pellet fraction, as previously described [35]. The primers used for cloning in pET32a are listed in Additional file 2: Table S4. The purified proteins were used to raise antibodies against $\alpha-\mathrm{SNAP}_{17224}$ and $\alpha-\mathrm{SNAP}_{16521}$ in rabbit, against $\alpha$-SNAP ${ }_{10856}$ in mouse and against GINSF in rat. All animal experiments adhered to the guidelines approved by the Institutional Animal Ethical Committee of Bose Institute (IAEC/BI/37/2015).

\section{Immunofluorescence and quantitative colocalization analyses}

Immunofluorescence was performed in trophozoites, encysting trophozoites (time of encystation indicated in respective figures) and cysts as previously described [40]. Briefly, cells were harvested by chilling the culture tubes on ice, followed by centrifugation at $1000 \times g$ for $10 \mathrm{~min}$. After washing with $1 \times$ PBS, the cells were fixed with $4 \%$ formaldehyde for $20 \mathrm{~min}$ at room temperature (RT). Following fixation, cells were harvested by centrifugation and treated with $0.1 \mathrm{M}$ glycine for $5 \mathrm{~min}$ at RT. Subsequently, trophozoites and encysting trophozoites were permeabilized with $0.1 \%$ Triton X-100 in $1 \times$ PBS (v/v) for $15 \mathrm{~min}$, followed by blocking with $2 \%$ BSA for $2 \mathrm{~h}$ at RT. Cysts were permeabilized with $0.2 \%$ Triton X-100 and $0.1 \%$ SDS for $15 \mathrm{~min}$, before blocking with $2 \%$ BSA. For labeling of all the four giardial proteins, the respective primary antisera were used at 1:50 dilution in $0.2 \%$ BSA and incubated for overnight at $4{ }^{\circ} \mathrm{C}$, with shaking. The following day, cells were washed thrice with $1 \times$ PBS and incubated with 1:400 dilution of any combination of the following secondary antibodies, as per requirement: Alexa Fluor 488-conjugated goat anti-rabbit, Alexa Fluor 488-conjugated goat anti-mouse, Alexa Fluor 594-conjug ated goat anti-mouse, Alexa Fluor 594-conjugated goat anti-rabbit and Alexa Fluor 488-conjugated goat anti-rat. All secondary antibodies were procured from Abcam (Cambridge, UK). Before washing away secondary antibodies, cells incubated with DAPI at $1 \mu \mathrm{g} / \mathrm{ml}$ concentration for $15 \mathrm{~min}$. Finally, cells washed three times with $1 \times$ PBS and resuspended in antifade medium (0.1\% $p$-phenylenediamine in $90 \%$ glycerol). Samples were imaged with the $63 \times$ objective of a confocal laser scanning microscope (Leica TCS SP8, Wetzlar, Germany). 3D deconvolution and colocalization analyses were performed with Leica Application Suit X and images were assembled with Adobe Photoshop CS3 and Adobe Illustrator CS3.

\section{Statistical analysis}

Statistical analyses for all the colocalization studies were performed with Leica Application Suit X (LAS X) software. The correlation coefficients, Pearson (Pr) and overlap (OC), values for each colocalization experiment were calculated on the basis of pixel-wise correlation between the signals emitted by the two fluorophores in each layer of six independent Z-stack. The Pr and OC values were plotted with GraphPad Prism 5.

\section{Real-time PCR}

cDNA preparation and real-time PCR was performed as previously described, with primers designed against unique regions of giardial $\alpha$-SNAPs (Additional file 2: Table S4) [35, 40]. PCR condition was as follows: initial denaturation at $95{ }^{\circ} \mathrm{C}$ for $5 \mathrm{~min}$, second denaturation at $95{ }^{\circ} \mathrm{C}$ for $30 \mathrm{~s}$, and annealing for $20 \mathrm{~s}$ at $56,64.5$ or 65.8 ${ }^{\circ} \mathrm{C}$ for $\mathrm{SNAP}_{17224}, \mathrm{SNAP}_{16521}$ and $\mathrm{SNAP}_{10856}$, respectively. The $C_{\mathrm{T}}$ values obtained for the three $\alpha$-SNAP genes were normalized against the expression of ribosomal protein S5 (GL50803_12981), while the expression of the CWP1 gene served as positive control for encystation $[37,46]$. Each experiment was performed in triplicate, with three technical replicates for each and data validation was done using two-tailed, paired t-test analysis in GraphPad Prism 5.

\section{Yeast two-hybrid assay}

Yeast two-hybrid assay was performed using full length NSF and $\alpha$-SNAPs of both yeast and Giardia, which were cloned in pGAD424 (prey vector having LEU2 marker) and pGBT9 (bait vector having TRP1 marker), respectively (Clonetech Laboratories, Mountain View, USA); the resulting fusion proteins had either the Gal4 activation domain (AD) or its DNA binding domain (BD), respectively (Additional file 2: Table S5) [74]. As per requirement, different pairs of the $\mathrm{AD}$ and $\mathrm{BD}$ constructs were co-transformed into the yeast strain PJ69-4A and the growth of each transformant, on YCM plates either lacking leucine, tryptophan and adenine 
(LTA) or lacking leucine, tryptophan and histidine, but having $2.5 \mathrm{mM}$ 3-AT (LTH 3-AT), was monitored following incubation at $30{ }^{\circ} \mathrm{C}$ for 2 to 3 days [75]. The binary interaction between the various NSF and $\alpha$-SNAP orthologues were also measured quantitatively by assessing the $\beta$-galactosidase activity by determining the nmol of o-nitrophenol formed from the hydrolysis of ONPG per min per mg of protein [76]. For this assay, experiments were performed in triplicate with two technical replicates for each sample. Results were statistically validated using a two-tailed, paired t-test in GraphPad Prism 5 software.

\section{Oxidative stress in Giardia}

Trophozoites were grown to confluency. The old medium was replaced with freshly-prepared medium and cells were allowed to grow for another $2 \mathrm{~h}$, prior to treatment with oxidative stress-inducing agents, either $150 \mu \mathrm{M} \mathrm{H}_{2} \mathrm{O}_{2}$ or $1 \mu \mathrm{g} / \mathrm{ml}$ metronidazole, for a period of $1 \mathrm{~h}$, at $37^{\circ} \mathrm{C}[45,46]$. To confirm intracellular ROS generation, cells were first harvested by chilling the tubes on ice, harvested by centrifugation at $1000 \times g$ for 10 min, washed thrice with warm PBS and treated with 2,7'-dichlorodihydrofluoresceine diacetate $\left(\mathrm{H}_{2} \mathrm{DCFDA}\right)$ (Sigma D6883, St. Louis, USA) at a concentration of 1.5 $\mu \mathrm{M}$, for 15 mins at $37{ }^{\circ} \mathrm{C}$ [45]. Finally, cells were fixed with $2 \%$ paraformaldehyde, washed thrice with PBS and observed under a confocal microscope.

\section{Additional files}

Additional file 1: Figure S1. Circular dichroism spectrum of giardial SNAPs. Far-UV circular dichroism spectra of the three giardial SNAPs, in 20 $\mathrm{mM}$ sodium phosphate buffer at $16^{\circ} \mathrm{C}$. Predicted helicity percentage, calculated by using the BeStSel server (bestsel.elte.hu/), is indicated in each spectrum. Percent helicity of Sec17, calculated on the basis of the crystal structure of Sec17 (1QQE), is 73\%. Figure S2. Expression of the putative SNAPs during the life cycle of Giardia. The expression of the three putative SNAP orthologues of Giardia were determined by reverse transcriptase PCR, using the CDNA prepared from trophozoites, encysting cells and cysts. The primers used for this analysis are given in Additional file 2: Table S4. PCR products were visualized on $1.2 \%$ agarose gel. The length of all the PCR products for each SNAP correspond to the expected size. Figure S3. Specificities of the antibodies for giardial a-SNAPs and NSF. a Western blot with trophozoite extract and each of the three purified giardial a-SNAPs. Expression and purification of the proteins have been described in the Experimental Procedures section. The 6 xHis-tag was removed from all three proteins prior to western blotting. The upper blot was incubated with anti-a-SNAP 17224 antibody while the middle and lower blots were incubated with anti-a-SNAP ${ }_{16521}$ and anti-a-SNAP 10856 antibodies, respectively. All antibodies were diluted 1000x prior to use. The presence of a $\sim 34 \mathrm{kDa}$ band in both the giardial extract and the corresponding overexpressed protein fraction in each blot indicates the specificity of that particular antibody. $\mathbf{b}$ Western blot with anti-GINSF antibody using extracts of Giardia trophozoites and E. coli overexpressing GINSF. Instead of the full-length protein, a stretch of 200-amino acids from the N-terminal segment of GINSF was tagged with the 6 xHis tag and expressed in $E$. coli. The expected size of this overexpressed protein is $39 \mathrm{kDa}$, while the size of the full-length GINSF is $91 \mathrm{kDa}$. Figure $\mathbf{S 4}$. Localization of a-SNAP 10856 to peripheral vesicles. a Colocalization of a-SNAP ${ }_{10856}$ with Lucifer yellow, a fluorescent dye that specifically stains acidic compartments, and, $\mathbf{b}$ a giardial PX domain-containing protein encoded by ORF GL50803_16548 and known to localize to the peripheral vesicles indicates that $\mathrm{a}-\mathrm{SNAP}{ }_{10856}$ localizes to the peripheral vesicles of trophozoites. The scattergram in each row indicates the analysis of colocalization between the two fluorophores, over the entire z-stack, by considering all the pixels within the whole area occupied by that cell. The values for Pearson correlation coefficient (Pr) and overlap coefficient $(\mathrm{OC})$ are indicated within the respective scattergrams. The intensity plot at the right in the lower panel indicates changes in fluorescence intensity of the red and green signals across the dotted white line drawn across the ROI. Scale-bars: $\mathbf{a}, \mathbf{b}, 5 \mu \mathrm{m}$. Figure $\mathbf{5 5}$. Immunolocalization of a-SNAP 17224 and a-SNAP 16521 to various subcellular structures. a Localization of a-SNAP ${ }_{17224}$ at the perinuclear region persisted after 30 $\mathrm{h}$ of encystation. The caret marks the perinuclear region. $\mathbf{b}$ a-SNAP 16521 is located both at the peripheral vesicles (PV) and the paraflagellar dense rods (PDRs) of different flagella at $1.5 \mathrm{~h}$ post induction of encystation. However, at $4 \mathrm{~h}$ post induction, a-SNAP 16521 is exclusively present at the PDRs, with no signal at the PVs. The triangle and arrow heads indicate PVs and the PDRs of different flagella, respectively. $\mathbf{c}$ Colocalization of a-tubulin (red) with a-SNAP ${ }_{16521}$ (green) in 16 h encysting trophozoites. The red and green signals are juxtaposed to each other at the anterior flagella, without any significant overlap. Cells were visualized with Alexa Fluor 488 and Cy5-conjugated secondary antibodies that bind to rabbit anti-a-SNAP 16521 and mouse anti a-tubulin, respectively. $\mathbf{d}$ Two individual zsections showing localization to the ventral disc periphery (left panel) and the PDRs (right panel). Scale-bars: a, b, c, d, $5 \mu \mathrm{m}$. Figure S6. Colocalization of a-SNAP 10856 and a PX-domain containing protein in cysts. Both a-SNAP 10856 and a PX-domain containing protein (GL50803_16548), a known lipid-interactor that localizes to vesicles, colocalize to cytoplasmic puncta in cysts. Carets mark some of the regions where there is considerable overlap of the red and green signals. The lower panels show regions of colocalization across the entire z-stack, with the left panel showing colocalization of the green signal (a-SNAP ${ }_{10856}$ ) on red (GL50803_16548) and the right panel shows the opposite, i.e. red on green. The intensity plot at the extreme right indicates changes in fluorescence intensity of the red and green signals across the dotted white line in the ROI. The values for Pearson (Pr) and overlap (OC) coefficient are given in the scatter plot. Scale-bar. $5 \mu \mathrm{m}$. Figure S7. Localization of GINSF and a-SNAP ${ }_{16521}$. Localization of GINSF (green) at the brush border (BB) and a-SNAP ${ }_{16521}$ (red) at the paraflagellar dense rods (PDRs) in $48 \mathrm{~h}$ encysting trophozoites. Each of the two fluorescent images has been individually merged with the DIC image to show the distribution of the fluorescent signals w.r.t. the flagella. Scale-bar: $5 \mu \mathrm{m}$. Figure S8. Detection of intracellular reactive oxygen species (ROS) by $\mathrm{H}_{2} \mathrm{DCFDA}$. Giardia trophozoites were treated with either $150 \mu \mathrm{M} \mathrm{H}_{2} \mathrm{O}_{2}$ or $1 \mu \mathrm{g} / \mathrm{ml}$ metronidazole for $1 \mathrm{~h}$ and intracellular ROS generation was monitored using $\mathrm{H}_{2}$ DCFDA. The top row shows untreated cells with no fluorescence signal of DCF. Green fluorescence signal was observed in cells exposed to oxidative stress, with either $\mathrm{H}_{2} \mathrm{O}_{2}$ (middle row) or metronidazole (bottom row) due to the formation of reactive oxygen species.

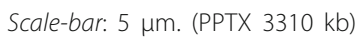

Additional file 2: Table S1. a-SNAP orthologues throughout the eukaryotes. Table S2. $\gamma$-SNAP orthologues throughout the eukaryotes. Table S3. SNARE binding residues of bovine a-SNAP and residues at analogous positions on Sec17 and giardial SNAPs. Table S4. Primer sequences. Table S5. List of constructs. Table S6. Percentage of cells exhibiting relocalization of the various a-SNAPs in different stages of encystation. Table S7. Percentage of cells exhibiting relocalization of the various a-SNAPs under oxidative stress. (DOCX $43 \mathrm{~kb}$ )

Additional file 3: Figure S9. Total number of Giardia cells with desired localization of three different a-SNAPs (a-SNAP17224 and a-SNAP10856 at the PVs, and a-SNAP16521 at the PDRs of different flagella) under, (a) $\mathrm{H}_{2} \mathrm{O}_{2}(150 \mu \mathrm{M})$ or $(\mathbf{b})$ metronidazole $(1 \mu \mathrm{g} / \mathrm{ml})$ stress were counted from five different fields. The "banned" sign indicates cells that were excluded from the final count, either due to absence of signal or because the cells were deformed. Cells where a given a-SNAP localized to the region other 
than the desired one, were marked with "cross" sign. Scale-bar: $25 \mu \mathrm{m}$. Figure S10. Total number of Giardia cells with desired localization of three different a-SNAPs (a-SNAP17224 and a-SNAP10856 at the PVs, and a-SNAP16521 at the PDRs of different flagella) under, (a) $\mathrm{H}_{2} \mathrm{O}_{2}(150 \mu \mathrm{M})$ or (b) metronidazole $(1 \mathrm{\mu g} / \mathrm{ml})$ stress were counted from five different fields. The "banned" sign indicates cells that were excluded from the final count, either due to absence of signal or because the cells were deformed. Cells where a given a-SNAP localized to the region other than the desired one, were marked with "cross" sign. Scale-bar: $25 \mu \mathrm{m}$. (PDF $14472 \mathrm{~kb}$ )

\section{Abbreviations}

3-AT: 3-amino-1,2,4-triazole; DAPI: 4', 6-diamidino-2-phenylindole; DIC: Differential interference contrast; GINSF: Giardia lamblia Nethylmaleimide sensitive factor; NSF: N-ethylmaleimide sensitive factor; ONPG: Ortho-nitrophenyl- $\beta$-D-galactopyranoside; ORF: Open reading frame; PBS: Phosphate-buffered saline; PDR: Paraflagellar dense rod; PM: Plasma membrane; ROS: Reactive oxygen species; RT: Room temperature; SNAP: Soluble NSF attachment protein; SNARE: Soluble NSF attachment protein receptor; YCM: Yeast complete medium

\section{Acknowledgments}

We acknowledge Dr A. K. Sil for his valuable suggestions during the course of this study and also for critically reading the manuscript. We thank Professor Randy Schekman and Professor Peter Novick for providing yeast strains. We acknowledge the Central Instrumentation Facility of Bose Institute, Kolkata, India, for DNA sequencing and confocal imaging and thank its staff members A. Poddar and S. Ghosh-Chakraborty for imaging and S. Biswas for CD spectroscopy. We are thankful to $\mathrm{Dr}$ A. Ghosh for helping with database searches and Dr A. Banerjee of Leica Microsystems for providing technical assistance during image processing. We acknowledge the assistance of D. Samanta and P. P. Dey of the Centre for Translational Animal Research, Bose Institute. We thank Professor P. Chakrabarti and Dr A. K. Sil for allowing us to use their laboratory facilities. We also thank members of the Sarkar Laboratory, Bose Institute, Kolkata, India, for proofreading the manuscript and providing valuable comments.

\section{Funding}

This study was supported by funds provided by the Bose Institute, Kolkata, India. SPD received fellowship support from the INSPIRE programme of the Department of Science and Technology, Government of India (IF120153).

\section{Availability of data and materials}

All data generated or analyzed during this study are included in this published article and its additional files.

\section{Authors' contributions}

SPD and SS conceived the study. SPD and AM carried out the experiment. KJ raised the antibodies. SG participated in the study design, provided critical inputs and helped draft the manuscript. SPD and SS analyzed the data and drafted the manuscript. All authors read and approved the final manuscript.

\section{Ethics approval and consent to participate}

All animal experiments were carried out under the approval of the Institutional Animal Ethics Committee, Bose Institute, Kolkata (IAEC/BI/37/2015).

\section{Consent for publication}

Not applicable.

\section{Competing interests}

The authors declare that they have no competing interests.

\section{Publisher's Note}

Springer Nature remains neutral with regard to jurisdictional claims in published maps and institutional affiliations.

\section{Author details}

'Department of Biochemistry, Bose Institute, P 1/12 CIT Road Scheme VII M, Kolkata, West Bengal 700054, India. ${ }^{2}$ Division of Molecular Medicine, Bose Institute, P 1/12 CIT Road Scheme VII M, Kolkata, West Bengal 700054, India. ${ }^{3}$ Present Address: Section on Developmental Genetics, Eunice Kennedy Shriver National Institute of Child Health and Human Development, NIH,
Bethesda, Maryland, USA. ${ }^{4}$ Division of Parasitology, National Institute of Cholera and Enteric Diseases, P-33, CIT Road, Scheme XM, Beliaghata, Kolkata, West Bengal 700010, India.

Received: 29 May 2018 Accepted: 13 September 2018

Published online: 04 October 2018

\section{References}

1. Dacks JB, Field MC. Evolution of the eukaryotic membrane-trafficking system: origin, tempo and mode. J Cell Sci. 2007;120:2977-85.

2. Lujan HD, Svärd S. Giardia: A Model Organism. New York: Springer; 2011

3. Lanfredi-Rangel A, Attias M, de Carvalho TM, Kattenbach WM, De Souza W. The peripheral vesicles of trophozoites of the primitive protozoan Giardia lamblia may correspond to early and late endosomes and to lysosomes. J Struct Biol. 1998;123:225-35.

4. Cooper GM, Hausman RE. The Cell - A Molecular Approach. 3rd ed. Washington DC: ASM Press; 2004

5. Alberts B, Johnson A, Lewis J, Morgan D, Raff M, Roberts K, Walter P. Molecular Biology of the Cell. 6th ed. New York: Garland Science; 2014.

6. Bonifacino JS, Glick BS. The mechanisms of vesicle budding and fusion. Cell. 2004;116:153-66.

7. Cai H, Reinisch K, Ferro-Novick S. Coats, tethers, Rabs, and SNAREs work together to mediate the intracellular destination of a transport vesicle. Dev Cell. 2007;12:671-82

8. Hong W. SNAREs and traffic. Biochim Biophys Acta. 1744;2005:493-517.

9. Söllner T, Whiteheart SW, Brunner M, Erdjument-Bromage H, Geromanos S, Tempst $P$, et al. SNAP receptors implicated in vesicle targeting and fusion. Nature. 1993;362:318-24.

10. Koumandou VL, Klute MJ, Herman EK, Nunez-Miguel R, Dacks JB, Field MC. Evolutionary reconstruction of the retromer complex and its function in Trypanosoma brucei. J Cell Sci. 2011;124:1496-509.

11. Touz MC, Rivero MR, Miras SL, Bonifacino JS. Lysosomal protein trafficking in Giardia lamblia: common and distinct features. Front Biosci Elite Ed. 2012;4: 1898-909

12. Langford TD, Silberman JD, Weiland ME-L, Svärd SG, McCaffery JM, Sogin ML, et al. Giardia lamblia: identification and characterization of Rab and GDI proteins in a genome survey of the ER to Golgi endomembrane system. Exp Parasitol. 2002;101:13-24

13. Marti M, Regös A, Li Y, Schraner EM, Wild P, Müller N, et al. An ancestral secretory apparatus in the protozoan parasite Giardia intestinalis. J Biol Chem. 2003;278:24837-48.

14. Saito-Nakano Y, Loftus BJ, Hall N, Nozaki T. The diversity of Rab GTPases in Entamoeba histolytica. Exp Parasitol. 2005;1 10:244-52.

15. Lal K, Field MC, Carlton JM, Warwicker J, Hirt RP. Identification of a very large Rab GTPase family in the parasitic protozoan Trichomonas vaginalis. Mol Biochem Parasitol. 2005:143:226-35.

16. Leung KF, Dacks JB, Field MC. Evolution of the multivesicular body ESCRT machinery; retention across the eukaryotic lineage. Traffic. 2008:9:1698-716.

17. Saha N, Dutta S, Datta SP, Sarkar S. The minimal ESCRT machinery of Giardia lamblia has altered inter-subunit interactions within the ESCRT-II and ESCRTIII complexes. Eur J Cell Biol. 2018;97:44-62.

18. Clary DO, Griff IC, Rothman JE. SNAPs, a family of NSF attachment proteins involved in intracellular membrane fusion in animals and yeast. Cell. 1990; 61:709-21.

19. Whiteheart SW, Griff IC, Brunner M, Clary DO, Mayer T, Buhrow SA, et al. SNAP family of NSF attachment proteins includes a brain-specific isoform. Nature. 1993;362:353-5.

20. Whiteheart SW, Kubalek EW. SNAPs and NSF: general members of the fusion apparatus. Trends Cell Biol. 1995:5:64-8.

21. Apodaca G, Cardone MH, Whiteheart SW, DasGupta BR, Mostov KE. Reconstitution of transcytosis in SLO-permeabilized MDCK cells: existence of an NSF-dependent fusion mechanism with the apical surface of MDCK cells. EMBO J. 1996;15:1471-81.

22. Miao Y, Miner C, Zhang L, Hanson PI, Dani A, Vig M. An essential and NSF independent role for a-SNAP in store-operated calcium entry. eLife. 2013;2: e00802.

23. Hanley JG, Khatri L, Hanson PI, Ziff EB. NSF ATPase and alpha-/beta-SNAPs disassemble the AMPA receptor-PICK1 complex. Neuron. 2002;34:53-67.

24. Morgan A, Burgoyne RD. A role for soluble NSF attachment proteins (SNAPs) in regulated exocytosis in adrenal chromaffin cells. EMBO J. 1995; 14(2):232-9. 
25. Peter F, Wong SH, Subramaniam VN, Tang BL, Hong W. Alpha-SNAP but not gamma-SNAP is required for ER-Golgi transport after vesicle budding and the Rab1-requiring step but before the EGTA-sensitive step. J Cell Sci. 1998; 111(Pt 17):2625-33.

26. Rivero FD, Müller D, Lujan HD. Secretory events during Giardia encystation. In: de Souza W, editor. Structures and Organelles in Pathogenic Protists. Microbiology Monographs, vol 17. Berlin-Heidelberg: Springer; 2010. p. 195-225.

27. Rice LM, Brunger AT. Crystal structure of the vesicular transport protein Sec17: implications for SNAP function in SNARE complex disassembly. Mol Cell. 1999;4:85-95.

28. Winter U, Chen X, Fasshauer D. A conserved membrane attachment site in alpha-SNAP facilitates N-ethylmaleimide-sensitive factor (NSF)-driven SNARE complex disassembly. J Biol Chem. 2009;284:31817-26.

29. Marz KE, Lauer JM, Hanson PI. Defining the SNARE complex binding surface of alpha-SNAP: implications for SNARE complex disassembly. J Biol Chem. 2003;278:27000-8.

30. Faso C, Bischof S, Hehl AB. The proteome landscape of Giardia lamblia encystation. PLoS One. 2013;8:e83207.

31. Sanderfoot AA, Assaad FF, Raikhel NV. The Arabidopsis genome. An abundance of soluble $\mathrm{N}$-ethylmaleimide-sensitive factor adaptor protein receptors. Plant Physiol. 2000;124:1558-69.

32. Kaiser CA, Schekman R. Distinct sets of SEC genes govern transport vesicle formation and fusion early in the secretory pathway. Cell. 1990;61:723-33.

33. Wampfler PB, Tosevski V, Nanni P, Spycher C, Hehl AB. Proteomics of secretory and endocytic organelles in Giardia lamblia. PLoS One. 2014;9:e94089.

34. Abodeely M, DuBois KN, Hehl A, Stefanic S, Sajid M, DeSouza W, et al. A contiguous compartment functions as endoplasmic reticulum and endosome/lysosome in Giardia lamblia. Eukaryot Cell. 2009;8:1665-76.

35. Jana A, Sinha A, Sarkar S. Phosphoinositide binding profiles of the PX domains of Giardia lamblia. Parasitol Int. 2017;66:606-14.

36. Elmendorf HG, Dawson SC, McCaffery JM. The cytoskeleton of Giardia lamblia. Int J Parasitol. 2003;33:3-28.

37. Mowatt MR, Luján HD, Cotten DB, Bowers B, Yee J, Nash TE, et al. Developmentally regulated expression of a Giardia lamblia cyst wall protein gene. Mol Microbiol. 1995;15:955-63.

38. Touz MC, Lujan HD, Hayes SF, Nash TE. Sorting of encystation-specific cysteine protease to lysosome-like peripheral vacuoles in Giardia lamblia requires a conserved tyrosine-based motif. J Biol Chem. 2003;278:6420-6.

39. Gaechter $V$, Schraner $E$, Wild $P$, Hehl AB. The single dynamin family protein in the primitive protozoan Giardia lamblia is essential for stage conversion and endocytic transport. Traffic. 2008;9:57-71.

40. Sinha A, Datta SP, Ray A, Sarkar S. A reduced WWA domain-containing proteasomal ubiquitin receptor of Giardia lamblia localizes to the flagellar pore regions in microtubule-dependent manner. Parasit Vectors. 2015;8:120.

41. Brown JR, Schwartz CL, Heumann JM, Dawson SC, Hoenger A. A detailed look at the cytoskeletal architecture of the Giardia lamblia ventral disc. J Struct Biol. 2016;194:38-48.

42. Kroetz MB, Hochstrasser M. Identification of SUMO-interacting proteins by yeast two-hybrid analysis. Methods Mol Biol. 2009;497:107-20

43. Lloyd D, Harris JC, Maroulis S, Biagini GA, Wadley RB, Turner MP, et al. The microaerophilic flagellate Giardia intestinalis: oxygen and its reaction products collapse membrane potential and cause cytotoxicity. Microbiol. 2000;146(Pt 12):3109-18.

44. Brown DM, Upcroft JA, Upcroft P. Free radical detoxification in Giardia duodenalis. Mol Biochem Parasitol. 1995;72:47-56.

45. Raj D, Ghosh E, Mukherjee AK, Nozaki T, Ganguly S. Differential gene expression in Giardia lamblia under oxidative stress: significance in eukaryotic evolution. Gene. 2014;535:131-9.

46. Ma'ayeh SY, Knörr L, Svärd SG. Transcriptional profiling of Giardia intestinalis in response to oxidative stress. Int J Parasitol. 2015;45:925-38.

47. Kienley N. Phylogenetic studies of the vesicular fusion machinery. PhD Thesis, Georg-August-Universität, Göttingen; 2010. https://d-nb.info/ $1042305226 / 34$

48. Elias EV, Quiroga R, Gottig N, Nakanishi H, Nash TE, Neiman A, et al. Characterization of SNAREs determines the absence of a typical Golgi apparatus in the ancient eukaryote Giardia lamblia. J Biol Chem. 2008;283: 35996-6010.

49. Chae TH, Kim S, Marz KE, Hanson PI, Walsh CA. The hyh mutation uncovers roles for alpha Snap in apical protein localization and control of neural cell fate. Nat Genet. 2004;36:264-70.
50. Andreeva AV, Kutuzov MA, Voyno-Yasenetskaya TA. A ubiquitous membrane fusion protein alpha SNAP: a potential therapeutic target for cancer, diabetes and neurological disorders? Expert Opin Ther Targets. 2006;10:723-33.

51. Li P, Miao Y, Dani A, Vig M. a-SNAP regulates dynamic, on-site assembly and calcium selectivity of Orai1 channels. Mol Biol Cell. 2016;27:2542-53.

52. Nakajima K, Hirose H, Taniguchi M, Kurashina H, Arasaki K, Nagahama M, et al. Involvement of BNIP1 in apoptosis and endoplasmic reticulum membrane fusion. EMBO J. 2004;23:3216-26.

53. Abel ES, Davids BJ, Robles LD, Loflin CE, Gillin FD, Chakrabarti R. Possible roles of protein kinase $A$ in cell motility and excystation of the early diverging eukaryote Giardia lamblia. J Biol Chem. 2001;276:10320-9.

54. Lauwaet T, Davids BJ, Torres-Escobar A, Birkeland SR, Cipriano MJ, Preheim SP, et al. Protein phosphatase 2A plays a crucial role in Giardia lamblia differentiation. Mol Biochem Parasitol. 2007;152:80-9.

55. Manning G, Reiner DS, Lauwaet T, Dacre M, Smith A, Zhai Y, et al. The minimal kinome of Giardia lamblia illuminates early kinase evolution and unique parasite biology. Genome Biol. 2011;12:R66.

56. Quarmby LM, Mahjoub MR. Caught Nek-ing: cilia and centrioles. J Cell Sci. 2005;118:5161-9.

57. Fry AM, O'Regan L, Sabir SR, Bayliss R. Cell cycle regulation by the NEK family of protein kinases. J Cell Sci. 2012;125:4423-33.

58. Parker JDK, Bradley BA, Mooers AO, Quarmby LM. Phylogenetic analysis of the Neks reveals early diversification of ciliary-cell cycle kinases. PLoS One. 2007;2:e1076.

59. Smith AJ, Lauwaet T, Davids BJ, Gillin FD. Giardia lamblia Nek1 and Nek2 kinases affect mitosis and excystation. Int J Parasitol. 2012:42:411-9.

60. Gaillard AR, Diener DR, Rosenbaum JL, Sale WS. Flagellar radial spoke protein 3 is an A-kinase anchoring protein (AKAP). J Cell Biol. 2001;153:443-8.

61. Lenaghan SC, Davis CA, Henson WR, Zhang Z, Zhang M. High-speed microscopic imaging of flagella motility and swimming in Giardia lamblia trophozoites. Proc Natl Acad Sci USA. 2011;108:E550-8.

62. Zhang Y. I-TASSER server for protein $3 D$ structure prediction. BMC Bioinformatics. 2008:9:40.

63. Kelley LA, Mezulis S, Yates CM, Wass MN, Sternberg MJ. The Phyre2 web portal for protein modelling, prediction and analysis. Nat Protoc. 2015;10: 845-58.

64. Thompson JD, Higgins DG, Gibson TJ. CLUSTAL W: improving the sensitivity of progressive multiple sequence alignment through sequence weighting, position-specific gap penalties and weight matrix choice. Nucleic Acids Res. 1994;22:4673-80.

65. Waterhouse AM, Procter JB, Martin DMA, Clamp M, Barton GJ. Jalview Version 2 - a multiple sequence alignment editor and analysis workbench. Bioinformatics. 2009:25:1189-91.

66. DeLano WL. The PyMOL Molecular Graphics System. DeLano Scientific LLC, Palo Alto, CA, USA. 2002. https://www.pymol.org. Accessed 21 Feb 2018.

67. Wheeler DL, Barrett T, Benson DA, Bryant SH, Canese K, Chetvernin V, et al. Database resources of the National Center for Biotechnology Information. Nucleic Acids Res. 2008:36:D13-21.

68. Aurrecoechea C, Barreto A, Basenko EY, Brestelli J, Brunk BP, Cade S, et al. EuPathDB: the eukaryotic pathogen genomics database resource. Nucleic Acids Res. 2017:45:D581-91.

69. UniProt Consortium T. UniProt: the universal protein knowledgebase. Nucleic Acids Res. 2018:46:2699.

70. Punta M, Coggill PC, Eberhardt RY, Mistry J, Tate J, Boursnell C, et al. The Pfam protein families database. Nucleic Acids Res. 2012:40:D290-301.

71. Kumar S, Stecher G, Tamura K. MEGA7: Molecular Evolutionary Genetics Analysis Version 7.0 for Bigger Datasets. Mol Biol Evol. 2016:33:1870-4.

72. Diamond LS, Harlow DR, Cunnick CC. A new medium for the axenic cultivation of Entamoeba histolytica and other Entamoeba. Trans R Soc Trop Med Hyg. 1978;72:431-2.

73. Boucher SE, Gillin FD. Excystation of in vitro-derived Giardia lamblia cysts. Infect Immun. 1990;58:3516-22.

74. Fields S, Song O. A novel genetic system to detect protein-protein interactions. Nature. 1989:340:245-6.

75. James P, Halladay J, Craig EA. Genomic libraries and a host strain designed for highly efficient two-hybrid selection in yeast. Genetics. 1996;144:1425-36.

76. Nielsen DA, Chou J, Mackrell AJ, Casadaban MJ, Steiner DF. Expression of a preproinsulin-beta-galactosidase gene fusion in mammalian cells. Proc Natl Acad Sci USA. 1983;80:5198-202. 